\title{
KRONROD EXTENSIONS WITH MULTIPLE NODES OF QUADRATURE FORMULAS FOR FOURIER COEFFICIENTS
}

\author{
GRADIMIR V. MILOVANOVIĆ AND MIODRAG M. SPALEVIĆ \\ Dedicated to the Memory of Professor Borislav Bojanov (1944-2009)
}

\begin{abstract}
We continue with analyzing quadrature formulas of high degree of precision for computing the Fourier coefficients in expansions of functions with respect to a system of orthogonal polynomials, started recently by Bojanov and Petrova [Quadrature formulae for Fourier coefficients, J. Comput. Appl. Math. 231 (2009), 378-391] and we extend their results. Construction of new Gaussian quadrature formulas for the Fourier coefficients of a function, based on the values of the function and its derivatives, is considered. We prove the existence and uniqueness of Kronrod extensions with multiple nodes of standard Gaussian quadrature formulas with multiple nodes for several weight functions, in order to construct some new generalizations of quadrature formulas for the Fourier coefficients. For the quadrature formulas for the Fourier coefficients based on the zeros of the corresponding orthogonal polynomials we construct Kronrod extensions with multiple nodes and highest algebraic degree of precision. For this very desirable kind of extension there do not exist any results in the theory of standard quadrature formulas.
\end{abstract}

\section{INTRODUCTION}

Let $\left\{P_{k}\right\}_{k=0}^{\infty}$ be a system of orthonormal polynomials on $[a, b]$ with respect to a weight function $\omega$ (integrable, non-negative function on $[a, b]$ that vanishes only at isolated points). The approximation of $f$ by the partial sums $S_{n}(f)$ of its series expansions

$$
f(x)=\sum_{k=0}^{\infty} a_{k}(f) P_{k}(x)
$$

with respect to a given system of orthonormal polynomials $\left\{P_{k}\right\}_{k=0}^{\infty}$ is a classical way of recovery of $f$. The numerical computation of the coefficients $a_{k}(f)$ in (1.1), i.e.,

$$
a_{k}(f)=\int_{a}^{b} \omega(t) P_{k}(t) f(t) d t
$$

Received by the editor February 1, 2012 and, in revised form, August 9, 2012 and September 4, 2012 .

2010 Mathematics Subject Classification. Primary 41A55; Secondary 65D30, 65D32.

Key words and phrases. Numerical integration, orthogonal polynomials, Gaussian quadratures, Fourier coefficients.

This work was supported in part by the Serbian Ministry of Education and Science (Research Projects: "Approximation of integral and differential operators and applications" (\#174015) \& "Methods of numerical and nonlinear analysis with applications" (\#174002)). 
requires the use of a quadrature formula. Evidently, an application of the $n$-point Gaussian quadrature formula with respect to the weight $\omega$ will give the exact result for all polynomials of degree at most $2 n-k-1, k<2 n-1$.

Following Bojanov and Petrova [3] (see also [2]) and using the same notation, we consider quadrature formulas of the type

$$
\int_{a}^{b} \omega(t) P_{k}(t) f(t) d t \approx \sum_{j=1}^{n} \sum_{i=0}^{\nu_{j}-1} c_{j i} f^{(i)}\left(x_{j}\right), \quad a<x_{1}<\cdots<x_{n}<b,
$$

where $\nu_{j}$ are given natural numbers (multiplicities) and $P_{k}(t)$ is a monic polynomial of degree $k$. A number $\ell$ is the algebraic degree of precision (ADP) of (1.3) if (1.3) is exact for all polynomials of degree $\ell$ and there is a polynomial of degree $\ell+1$ for which this formula is not exact. By $e(\nu)$ is denoted the smallest non-negative even integer $\geq \nu$ (clearly $e(\nu)=0$ for $\nu \leq 0)$, and by $\sigma\left(P_{k}\right)$ the number of zeros of $P_{k}$ in $(a, b)$ with odd multiplicities. It is easy to see that the ADP(1.3) does not exceed

$$
e\left(\nu_{1}-\tau_{1}\right)+\cdots+e\left(\nu_{n}-\tau_{n}\right)+\sigma\left(P_{k}\right)-1,
$$

since the formula is not exact for the polynomial

$$
\left(t-x_{1}\right)^{e\left(\nu_{1}-\tau_{1}\right)} \cdots\left(t-x_{n}\right)^{e\left(\nu_{n}-\tau_{n}\right)}\left(t-t_{1}\right) \cdots\left(t-t_{m}\right),
$$

where $m=\sigma\left(P_{k}\right), t_{1}, \ldots, t_{m} \in(a, b)$, are the zeros of $P_{k}$ with odd multiplicities, $\tau_{i}:=1$ if $x_{i} \in\left\{t_{1}, \ldots, t_{m}\right\}$ and $\tau_{i}:=0$ otherwise. Notice that in our applications the polynomial $P_{k}$ in formula (1.2) for $a_{k}(f)$ is the $k$-th orthogonal polynomial on $[a, b]$ with weight $\omega$; thus all its zeros are with multiplicity one and we have that $\sigma\left(P_{k}\right)=k$.

In [3], for the sake of convenience, Bojanov and Petrova defined the formula (1.3) to be Gaussian, if it has maximal ADP, that is, if

$$
\operatorname{ADP}(\underline{1.3})=e\left(\nu_{1}-\tau_{1}\right)+\cdots+e\left(\nu_{n}-\tau_{n}\right)+\sigma\left(P_{k}\right)-1 \text {. }
$$

A complete characterization of the Gaussian formulas of form (1.3) and explicit construction of such formulas in several particular cases is given in [3].

The paper is organized as follows. In Section 2 we repeat general remarks concerning standard Gaussian quadrature formulas with multiple nodes, since the study and numerical construction of formulas of type (1.3), and their generalizations, for Fourier coefficients can be reduced to the study and numerical construction of standard multiple node quadratures. In Subsection 2.1 the conditions of existence of general real Kronrod extensions with multiple nodes of standard Gaussian quadrature formulas with multiple nodes are given. If these extensions exist, then they are uniquely determined. In Subsection 2.2 we prove the existence and uniqueness of general real Kronrod extensions with multiple nodes of some standard Gaussian quadrature formulas with multiple nodes for the generalized Chebyshev and Gori-Micchelli weight functions, in order to construct some new generalizations of quadrature formulas for the Fourier coefficients by using the important theorem by Bojanov and Petrova [3, Theorem 2.1] (see also Theorem 2.2 below) which reveals the relation between the standard quadratures and the quadratures for Fourier coefficients. We finish Section 2 by adding some remarks concerning the computational aspects of the results by Bojanov and Petrova [3], and by extending their results from [3, Corollary 2.5] to the more general case. In Section 3 a connection to the Gauss-Kronrod and generalized averaged Gaussian quadrature formulas (all their nodes are simple) is presented. Finally, in Section 4 for the quadrature formulas for 
the Fourier coefficients (and for their generalizations) based on the zeros of the corresponding orthogonal polynomials we construct Kronrod extensions with multiple nodes and highest algebraic degree of precision. A numerical example is included. For this very desirable kind of extension there do not exist any results in the theory of standard quadrature formulas.

\section{General observations and numerical COnstruction}

Let

$$
\pi_{n}(\mathbb{R}):=\left\{P(t): P(t)=\sum_{k=0}^{n} d_{k} t^{k}, d_{k} \in \mathbb{R}\right\}
$$

represent the space of all polynomials in one variable of degree at most $n$. Bojanov and Petrova [3, Section 2] discuss general remarks concerning Gaussian quadrature formulas with multiple nodes, since the study of formulas of type (1.3) for Fourier coefficients can be reduced to the study of standard multiple node quadratures. We repeat the following theorem established by Ghizzetti and Ossicini [15].

Theorem 2.1. For any given set of odd multiplicities $\nu_{1}, \ldots, \nu_{n}\left(\nu_{j}=2 s_{j}+1, s_{j} \in\right.$ $\left.\mathbb{N}_{0}, j=1, \ldots, n\right)$, there exists a unique quadrature formula of the form

$$
\int_{a}^{b} \omega(t) f(t) d t \approx \sum_{j=1}^{n} \sum_{i=0}^{\nu_{j}-1} a_{j i} f^{(i)}\left(x_{j}\right), \quad a \leq x_{1}<\cdots<x_{n} \leq b,
$$

of $\mathrm{ADP}=\nu_{1}+\cdots+\nu_{n}+n-1$, which is the well known Chakalov-Popoviciu quadrature formula (see [5], 38]). The nodes $x_{1}, \ldots, x_{n}$ of this quadrature are determined uniquely by the orthogonality property

$$
\int_{a}^{b} \omega(t) \prod_{k=1}^{n}\left(t-x_{k}\right)^{\nu_{k}} Q(t) d t=0, \quad \forall Q \in \pi_{n-1}(\mathbb{R}) .
$$

The corresponding (monic) orthogonal polynomial $\prod_{k=1}^{n}\left(t-x_{k}\right)$ is known in the classical literature as a $\sigma$-orthogonal polynomial, with $\sigma=\sigma_{n}=\left(s_{1}, \ldots, s_{n}\right)$, where $n$ indicates the size of the array.

Quadratures of type (2.1) with equal multiplicities $\nu_{1}=\cdots=\nu_{n}=\nu$, with $\nu$ being an odd number $(\nu=2 s+1, s \in \mathbb{N})$, have been studied by Turán [44. In this case, the Gaussian quadrature is called the Gauss-Turán quadrature of type $\nu(=2 s+1)$, and the corresponding (monic) orthogonal polynomial $\prod_{k=1}^{n}\left(t-x_{k}\right)$ is called an $s$-orthogonal polynomial.

Bojanov and Petrova [3] describe the connection between quadratures with multiple nodes and formulas of type (1.3). For the system of nodes $\mathbf{x}:=\left(x_{1}, \ldots, x_{n}\right)$ with corresponding multiplicities $\bar{\nu}:=\left(\nu_{1}, \ldots, \nu_{n}\right)$, they define the polynomials

$$
\Lambda(t ; \mathbf{x}):=\prod_{m=1}^{n}\left(t-x_{m}\right), \quad \Lambda_{j}(t ; \mathbf{x}):=\frac{\Lambda(t ; \mathbf{x})}{t-x_{j}}, \quad \Lambda^{\bar{\nu}}(t ; \mathbf{x}):=\prod_{m=1}^{n}\left(t-x_{m}\right)^{\nu_{m}}
$$

set $x_{j}^{\nu_{j}}:=\left(x_{j}, \ldots, x_{j}\right)\left[x_{j}\right.$ repeats $\nu_{j}$ times $], j=1, \ldots, n$, denote by $g\left[x_{1}, \ldots, x_{m}\right]$ the divided difference of $g$ at the points $x_{1}, \ldots, x_{m}$, and state and prove the following important theorem which reveals the relation between the standard quadratures and the quadratures for Fourier coefficients. 
Theorem 2.2. For any given sets of multiplicities $\bar{\mu}:=\left(\mu_{1}, \ldots, \mu_{k}\right)$ and $\bar{\nu}:=$ $\left(\nu_{1}, \ldots, \nu_{n}\right)$, and nodes $y_{1}<\cdots<y_{k}, x_{1}<\cdots<x_{n}$, there exists a quadrature formula of the form

$$
\int_{a}^{b} \omega(t) \Lambda^{\bar{\mu}}(t ; \mathbf{y}) f(t) d t \approx \sum_{j=1}^{n} \sum_{i=0}^{\nu_{j}-1} c_{j i} f^{(i)}\left(x_{j}\right),
$$

with $\mathrm{ADP}=N$ if and only if there exists a quadrature formula of the form

$$
\int_{a}^{b} \omega(t) f(t) d t \approx \sum_{m=1}^{k} \sum_{\lambda=0}^{\mu_{m}-1} b_{m \lambda} f^{(\lambda)}\left(y_{m}\right)+\sum_{j=1}^{n} \sum_{i=0}^{\nu_{j}-1} a_{j i} f^{(i)}\left(x_{j}\right)
$$

which has degree of precision $N+\mu_{1}+\cdots+\mu_{k}$. In the case $y_{m}=x_{j}$ for some $m$ and $j$, the corresponding terms in both sums combine in one term of the form:

$$
\sum_{\lambda=0}^{\mu_{m}+\nu_{j}-1} d_{m \lambda} f^{(\lambda)}\left(y_{m}\right)
$$

Let us suppose that the coefficients $a_{j i}\left(j=1, \ldots, n ; i=0, \ldots, \nu_{j}-1\right)$ in (2.3) are known. By acting as in the first part of the proof of Theorem 2.1 in [3] we can determine the coefficients $c_{j i}\left(j=1, \ldots, n ; i=0, \ldots, \nu_{j}-1\right)$ in (2.2). Namely, applying (2.3) to the polynomial $\Lambda^{\bar{\mu}}(\cdot ; \mathbf{y}) f$, where $f \in \pi_{N}(\mathbb{R})$, the first sum in (2.3) vanishes and we can obtain (see [3, Eq. (2.4)]):

$$
\begin{aligned}
\int_{a}^{b} \omega(t) \Lambda^{\bar{\mu}}(t ; \mathbf{y}) f(t) d t & =\sum_{j=1}^{n}\left(\left.\sum_{i=0}^{\nu_{j}-1} a_{j i}\left[\Lambda^{\bar{\mu}}(t ; \mathbf{y}) f(t)\right]^{(i)}\right|_{t=x_{j}}\right) \\
& =\sum_{j=1}^{n} \sum_{i=0}^{\nu_{j}-1} c_{j i} f^{(i)}\left(x_{j}\right),
\end{aligned}
$$

where

$$
c_{j i}=\left.\sum_{s=i}^{\nu_{j}-1} a_{j s}\left(\begin{array}{l}
s \\
i
\end{array}\right)\left[\Lambda^{\bar{\mu}}(t ; \mathbf{y})\right]^{(s-i)}\right|_{t=x_{j}}\left(j=1,2, \ldots, n ; i=0,1, \ldots, \nu_{j}-1\right) .
$$

2.1. General real Kronrod extensions of Chakalov-Popoviciu quadratures. Numerically stable methods for constructing nodes $x_{j}$ and coefficients $a_{j i}$ in GaussTurán and Chakalov-Popoviciu quadrature formulas with multiple nodes can be found in [16], 12], 28], [43]. For the asymptotic representation of the coefficients $a_{j i}$ see 35 . Some interesting results concerning this theory and its applications can be found in [25], 42, and references therein, and [14, [21], 35]. The remainder term for these quadratures, when the integrand is an analytic function, has been analyzed in [26, 29, 27, 30, 31.

The following generalized Chebyshev weight functions $\omega(t)=\omega_{i}(t)$ will be of interest in this paper:
(a) $\omega_{1}(t)=\left(1-t^{2}\right)^{-1 / 2}$,
(c) $\omega_{3}(t)=(1-t)^{-1 / 2}(1+t)^{1 / 2+s}$,
(b) $\omega_{2}(t)=\left(1-t^{2}\right)^{1 / 2+s}$,
(d) $\omega_{4}(t)=(1-t)^{1 / 2+s}(1+t)^{-1 / 2}$.

The Chebyshev polynomials $T_{n}$ are $s$-orthogonal on $(-1,1)$ for each $s \geq 0$ (see Bernstein [1]). Ossicini and Rosati 34 found three other weight functions $\omega_{i}(t)(i=$ $2,3,4)$ for which the $s$-orthogonal polynomials can be identified as the Chebyshev 
polynomials of the second, third, and fourth kind: $U_{n}, V_{n}$, and $W_{n}$, which are defined by

$$
U_{n}(t)=\frac{\sin (n+1) \theta}{\sin \theta}, V_{n}(t)=\frac{\cos \left(n+\frac{1}{2}\right) \theta}{\cos (\theta / 2)}, W_{n}(t)=\frac{\sin \left(n+\frac{1}{2}\right) \theta}{\sin (\theta / 2)},
$$

respectively (cf. 9] and [13), where $t=\cos \theta$. However, these weight functions depend on $s$ (see (b), (c), (d)). It is easy to see that $W_{n}(-t)=(-1)^{n} V_{n}(t)$, so that in the investigation it is sufficient to study $\omega_{1}(t), \omega_{2}(t)$ and one of either $\omega_{3}(t)$ or $\omega_{4}(t)$.

For each $n \in \mathbb{N}$, Gori and Micchelli 17 introduced an interesting class of weight functions defined on $[-1,1]$ for which explicit Gauss-Turán quadrature formulas of all orders can be found. In other words, these classes of weight functions have the peculiarity that the corresponding $s$-orthogonal polynomials, $s \in \mathbb{N}$, of the same degree, are independent of $s$. This class includes certain generalized Jacobi weight functions $\omega_{n, \mu}(t)=\left|U_{n-1}(t) / n\right|^{2 \mu+1}\left(1-t^{2}\right)^{\mu}$, where $U_{n-1}(\cos \theta)=\sin n \theta / \sin \theta$ (Chebyshev polynomial of the second kind) and $\mu>-1$. In this case, the Chebyshev polynomials $T_{n}$ appear as $s$-orthogonal polynomials, $s \in \mathbb{N}$.

Let $\sigma^{*}=\sigma_{m}^{*}=\left(s_{1}^{*}, s_{2}^{*}, \ldots, s_{m}^{*}\right)\left(s_{\mu}^{*} \in \mathbb{N}_{0}, \mu=1,2, \ldots, m\right)$. Following the wellknown idea of Kronrod [20], 8] (see also [32, [33], 24]), we extend a formula of type (2.1),

$$
\int_{a}^{b} f(t) \omega(t) d t \approx \sum_{\nu=1}^{n} \sum_{i=0}^{2 s_{\nu}} a_{\nu i} f^{(i)}\left(x_{\nu}\right)
$$

where $a \leq x_{1}<x_{2}<\cdots<x_{n} \leq b$, to the interpolatory quadrature formula

$$
\int_{a}^{b} f(t) \omega(t) d t \approx \sum_{\nu=1}^{n} \sum_{i=0}^{2 s_{\nu}} b_{\nu i} f^{(i)}\left(x_{\nu}\right)+\sum_{\mu=1}^{m} \sum_{j=0}^{2 s_{\mu}^{*}} c_{\mu j}^{*} f^{(j)}\left(x_{\mu}^{*}\right)
$$

where $x_{\nu}$ are the same nodes as in (2.5), and the new nodes $x_{\mu}^{*}$ and new weights $b_{\nu i}, c_{\mu j}^{*}$ are chosen to maximize the degree of precision of (2.6) which is greater than or equal to

$$
\sum_{\nu=1}^{n}\left(2 s_{\nu}+1\right)+\sum_{\mu=1}^{m}\left(2 s_{\mu}^{*}+1\right)+m-1=2\left(\sum_{\nu=1}^{n} s_{\nu}+\sum_{\mu=1}^{m} s_{\mu}^{*}\right)+n+2 m-1 .
$$

We call the quadrature formula (2.6) Chakalov-Popoviciu-Kronrod quadrature formula. A particular case of this formula is the Gauss-Turán-Kronrod quadrature formula, if $s_{1}=s_{2}=\cdots=s_{n}=s$.

The well-known Gauss-Kronrod quadrature formula, if $s_{1}=s_{2}=\cdots=s_{n}=$ $0, s_{1}^{*}=s_{2}^{*}=\cdots=s_{m}^{*}=0$, and $m=n+1$, is a particular case of both justmentioned quadrature formulae. In the theory of Gauss-Kronrod quadrature formulas, the Sieltjes polynomials $E_{n+1}(t)$, whose zeros are the nodes $x_{\mu}^{*}$, namely $E_{n+1}(t) \equiv E_{n+1}(t, \omega):=\prod_{\mu=1}^{n+1}\left(t-x_{\mu}^{*}\right)$, play an important role. Also, of foremost interest are weight functions for which the Gauss-Kronrod quadrature formula has the property that:

(i) All $n+1$ nodes $x_{\mu}^{*}$ are in $(a, b)$ and are simple (i.e., that all zeros of the Stieltjes polynomial $E_{n+1}(t)$ are in $(a, b)$ and are simple).

Also, desirable are weight functions which have, in addition to (i), the following properties: 
(ii) The interlacing property. Namely, that the nodes $x_{\mu}^{*}$ and $x_{\nu}$ separate each other (i.e., the $n+1$ zeros of $E_{n+1}(t)$ separate the $n$ zeros of the orthogonal polynomial $\left.\prod_{\nu=1}^{n}\left(t-x_{\nu}\right)\right)$; and

(iii) all quadrature weights are positive.

On the basis of the above facts, it seems it is most natural to consider ChakalovPopoviciu-Kronrod quadratures (2.6) in which $m=n+1$, i.e.,

$$
\int_{a}^{b} f(t) \omega(t) d t \approx \sum_{\nu=1}^{n} \sum_{i=0}^{2 s_{\nu}} b_{\nu i} f^{(i)}\left(x_{\nu}\right)+\sum_{\mu=1}^{n+1} \sum_{j=0}^{2 s_{\mu}^{*}} c_{\mu j}^{*} f^{(j)}\left(x_{\mu}^{*}\right) .
$$

We know that in the general case of quadratures with multiple nodes not all quadrature weights have to be positive. Therefore, for Kronrod extensions of Gaussian quadrature formulas with multiple nodes we cannot consider the property (iii) as desirable.

On the other hand, it is desirable that the nodes $x_{\mu}^{*}, \mu=1, \ldots, n+1$, be all real, $x_{1}^{*}<x_{2}^{*}<\cdots<x_{n+1}^{*}$, as well as to satisfy the interlacing property, i.e.,

$$
x_{1}^{*}<x_{1}<x_{2}^{*}<x_{2}<\cdots<x_{n}<x_{n+1}^{*} .
$$

We are interested in the Chakalov-Popoviciu-Kronrod quadratures (2.7) in which the nodes $x_{\mu}^{*}, \mu=1, \ldots, n+1$, satisfy the property $x_{1}^{*}<x_{2}^{*}<\cdots<x_{n+1}^{*}$.

Proposition 2.3. Let the nodes be ordered. The interpolatory quadrature formula (2.7) with multiple nodes has degree of precision $2\left(\sum_{\nu=1}^{n} s_{\nu}+\sum_{\mu=1}^{n+1} s_{\mu}^{*}\right)+3 n+1$ if and only if the following orthogonality conditions:

$$
\int_{a}^{b} \prod_{\nu=1}^{n}\left(t-x_{\nu}\right)^{2 s_{\nu}+1} \prod_{\mu=1}^{n+1}\left(t-x_{\mu}^{*}\right)^{2 s_{\mu}^{*}+1} t^{k} \omega(t) d t=0, \quad k=0,1, \ldots, n,
$$

hold.

Proof. Let $\pi_{n}(t) \equiv \pi_{n, \sigma}(t)=\prod_{\nu=1}^{n}\left(t-x_{\nu}\right)$ be the $\sigma$-orthogonal polynomial based on the nodes $x_{\nu}$, and let $E_{n+1}^{\left(\sigma^{*}\right)}(t)=\prod_{\mu=1}^{n+1}\left(t-x_{\mu}^{*}\right)$ be the corresponding generalized Stieltjes polynomial based on the nodes $x_{\mu}^{*}$. The conditions (2.9) can be reinterpreted in the form

$$
\int_{a}^{b} E_{n+1}^{\left(\sigma^{*}\right)}(t) \pi_{n, \sigma}(t) t^{k} \widetilde{\omega}(t) d t=0, \quad k=0,1, \ldots, n,
$$

where $\widetilde{\omega}(t)=\omega(t) \prod_{\nu=1}^{n}\left(t-x_{\nu}\right)^{2 s_{\nu}} \prod_{\mu=1}^{n+1}\left(t-x_{\mu}^{*}\right)^{2 s_{\mu}^{*}}$ is the new implicitly given weight function (see Engels [7, pp. 214-226]). Therefore, because of (2.10), the generalized (monic) Stieltjes polynomial $E_{n+1}^{\left(\sigma^{*}\right)}(t)$ is uniquely determined (cf. [32, p. 145]).

Suppose now that the quadrature formula (2.7) has the degree of precision $2\left(\sum_{\nu=1}^{n} s_{\nu}+\sum_{\mu=1}^{n+1} s_{\mu}^{*}\right)+3 n+1$, and let

$$
f_{k}(t)=\prod_{\nu=1}^{n}\left(t-x_{\nu}\right)^{2 s_{\nu}+1} \prod_{\mu=1}^{n+1}\left(t-x_{\mu}^{*}\right)^{2 s_{\mu}^{*}+1} t^{k}, \quad k \in\{0,1, \ldots, n\} .
$$

Then, we have that

$$
\operatorname{deg}\left(f_{k}\right) \leq \sum_{\nu=1}^{n}\left(2 s_{\nu}+1\right)+\sum_{\mu=1}^{n+1}\left(2 s_{\mu}^{*}+1\right)+n=2\left(\sum_{\nu=1}^{n} s_{\nu}+\sum_{\mu=1}^{n+1} s_{\mu}^{*}\right)+3 n+1 .
$$


Let us determine, for instance, $f_{k}^{(i)}\left(x_{1}\right), i=0,1, \ldots, 2 s_{1}$. Using the following representation $f_{k}(t)=\left(t-x_{1}\right)^{2 s_{1}+1} u_{k}(t)$, where

$$
u_{k}(t)=\prod_{\nu=2}^{n}\left(t-x_{\nu}\right)^{2 s_{\nu}+1} \prod_{\mu=1}^{n+1}\left(t-x_{\mu}^{*}\right)^{2 s_{\mu}^{*}+1} t^{k}, \quad k \in\{0,1, \ldots, n\},
$$

and by using the Leibnitz's formula, we have

$$
f_{k}^{(i)}(t)=\sum_{\ell=0}^{i}\left(\begin{array}{l}
i \\
\ell
\end{array}\right) u_{k}^{(i-\ell)}(t)\left(\left(t-x_{1}\right)^{2 s_{1}+1}\right)^{(\ell)}, \quad i=0,1, \ldots, 2 s_{1} .
$$

Therefore, $f_{k}^{(i)}\left(x_{1}\right)=0, i=0,1, \ldots, 2 s_{1}$. In a similar way we conclude that

$$
\begin{aligned}
& f_{k}^{(i)}\left(x_{\nu}\right)=0 ; \quad i=0,1, \ldots, 2 s_{\nu}, \quad \nu=2, \ldots, n, \\
& f_{k}^{(i)}\left(x_{\mu}^{*}\right)=0 ; \quad i=0,1, \ldots, 2 s_{\mu}^{*}, \quad \mu=1, \ldots, n+1 .
\end{aligned}
$$

Using these facts, for the given functions $f_{k}$, we have that

$$
\sum_{\nu=1}^{n} \sum_{i=0}^{2 s_{\nu}} b_{\nu i} f_{k}^{(i)}\left(x_{\nu}\right)+\sum_{\mu=1}^{n+1} \sum_{j=0}^{2 s_{\mu}^{*}} c_{\mu j}^{*} f_{k}^{(j)}\left(x_{\mu}^{*}\right)=0 .
$$

Because of the latter, we conclude that (2.9) holds.

Now, let the orthogonality conditions (2.9) hold. Consider an arbitrary polynomial $g(t)$ of degree $\leq 2\left(\sum_{\nu=1}^{n} s_{\nu}+\sum_{\mu=1}^{n+1} s_{\mu}^{*}\right)+3 n+1$, which can be represented in the form

$$
g(t)=\prod_{\nu=1}^{n}\left(t-x_{\nu}\right)^{2 s_{\nu}+1} \prod_{\mu=1}^{n+1}\left(t-x_{\mu}^{*}\right)^{2 s_{\mu}^{*}+1} u_{n}(t)+v_{q}(t),
$$

where $u_{n}(t), v_{q}(t)$ are polynomials of the degrees $n, q$, respectively, and $q=$ $2\left(\sum_{\nu=1}^{n} s_{\nu}+\sum_{\mu=1}^{n+1} s_{\mu}^{*}+n\right)$. As an interpolatory quadrature formula (2.7) is exact for each polynomial of degree $\leq 2\left(\sum_{\nu=1}^{n} s_{\nu}+\sum_{\mu=1}^{n+1} s_{\mu}^{*}+n\right)$. Therefore, because of the last facts and (2.10),

$$
\begin{aligned}
\int_{a}^{b} g(t) \omega(t) d t= & \int_{a}^{b} \prod_{\nu=1}^{n}\left(t-x_{\nu}\right)^{2 s_{\nu}+1} \prod_{\mu=1}^{n}\left(t-x_{\mu}^{*}\right)^{2 s_{\mu}^{*}+1} u_{n}(t) \omega(t) d t \\
& +\int_{a}^{b} v_{q}(t) \omega(t) d t \\
= & \int_{a}^{b} v_{q}(t) \omega(t) d t=\sum_{\nu=1}^{n} \sum_{i=0}^{2 s_{\nu}} b_{\nu i} v_{q}^{(i)}\left(x_{\nu}\right)+\sum_{\mu=1}^{n+1} \sum_{j=0}^{2 s_{\mu}^{*}} c_{\mu j}^{*} v_{q}^{(j)}\left(x_{\mu}^{*}\right) .
\end{aligned}
$$

Because of $g^{(i)}\left(x_{\nu}\right)=v_{q}^{(i)}\left(x_{\nu}\right), \nu=1, \ldots, n, i=0,1, \ldots, 2 s_{\nu}$, and $g^{(j)}\left(x_{\mu}^{*}\right)=$ $v_{q}^{(j)}\left(x_{\mu}^{*}\right), \mu=1, \ldots, n+1, j=0,1, \ldots, 2 s_{\mu}^{*}$, the previous formula reduces to

$$
\int_{a}^{b} g(t) \omega(t) d t=\sum_{\nu=1}^{n} \sum_{i=0}^{2 s_{\nu}} b_{\nu i} g^{(i)}\left(x_{\nu}\right)+\sum_{\mu=1}^{n+1} \sum_{j=0}^{2 s_{\mu}^{*}} c_{\mu j}^{*} g^{(j)}\left(x_{\mu}^{*}\right),
$$


which means that the quadrature formula (2.7) has the degree of precision $2\left(\sum_{\nu=1}^{n} s_{\nu}+\sum_{\mu=1}^{n+1} s_{\mu}^{*}\right)+3 n+1$.

For the uniqueness of the Chakalov-Popoviciu-Kronrod quadrature formula (2.7) we have the following statement.

Proposition 2.4. If the interpolatory quadrature formula (2.7) with multiple nodes and degree of precision $2\left(\sum_{\nu=1}^{n} s_{\nu}+\sum_{\mu=1}^{n+1} s_{\mu}^{*}\right)+3 n+1$ exists, then it is unique.

Proof. Let us write the interpolatory quadrature formula of type (2.7) in the form

$$
\int_{a}^{b} f(t) \omega(t) d t \approx \sum_{\nu=1}^{2 n+1} \sum_{i=0}^{2 s_{\nu}} a_{\nu i} f^{(i)}\left(x_{\nu}\right)
$$

where $n$ of its nodes $x_{\nu}$ are fixed. It has a degree of precision equal to $N=$ $3 n+2 \sum_{\nu=1}^{2 n+1} s_{\nu}+1$ if and only if $K=2 n+1+2 \sum_{\nu=1}^{2 n+1} s_{\nu}$ coefficients $a_{\nu i}$ satisfy the following system of $N+1$ linear equations

$$
\sum_{\nu=1}^{2 n+1} \sum_{i=0}^{2 s_{\nu}} a_{\nu i} u_{j}^{(i)}\left(x_{\nu}\right)=\int_{a}^{b} u_{j}(t) \omega(t) d t, \quad j=0,1, \ldots, N
$$

where $u_{j}(t), j=0,1, \ldots, N$, are linearly independent functions from the space $\pi_{N}(\mathbb{R})$. Assume the matrix $\left[u_{j}^{(i)}\left(x_{\nu}\right)\right]$ with $N+1$ rows and $K$ columns has rank $N+1-q(q>1$ because $N>K)$. If all nodes are mutually different, this is equivalent to the following statement: The boundary differential problem

$$
\frac{d^{N+1} u}{d t^{N+1}}=0, u_{j}^{(i)}\left(x_{\nu}\right)=0 \quad\left(i=0,1, \ldots, 2 s_{\nu} ; \nu=1,2, \ldots, 2 n+1\right)
$$

has $q$ linearly independent solutions $U_{k}, k=0,1, \ldots, q-1$ (see [14, pgs. 41-43]).

The system (2.12) is consistent if and only if the $q$ conditions

$$
\int_{a}^{b} U_{k}(t) \omega(t) d t=0, \quad k=0,1, \ldots, q-1,
$$

are satisfied. In that case (2.12) has $\infty^{K-(N+1-q)}$ solutions. (We use the same notation as in [14].)

It is easy to see that the problem (2.13) has the following $n+1$ linearly independent nontrivial solutions

$$
t^{k} \prod_{\nu=1}^{2 n+1}\left(t-x_{\nu}\right)^{2 s_{\nu}+1}, \quad k=0,1, \ldots, n
$$

Denoting them as $U_{k}(t)$, the conditions (2.14) become the conditions (2.9). The system (2.12) has one and only one solution, because

$$
K-(N+1-q)=\left(2 n+1+2 \sum_{\nu=1}^{2 n+1} s_{\nu}\right)+(n+1)-\left(3 n+2 \sum_{\nu=1}^{2 n+1} s_{\nu}+2\right)=0 .
$$

The above results can be obtained for the more general case of the quadrature formula (2.7). Namely, for each node $x_{\mu}^{*} \notin(a, b)$ instead of the multiplicity $2 s_{\mu}^{*}+1$ the multiplicity $n_{\mu}^{*}+1\left(n_{\mu}^{*} \in \mathbb{N}_{0}\right)$ can be considered, since the factor $\left(t-x_{\mu}^{*}\right)^{n_{\mu}^{*}+1}$ does not change sign on $(-1,1)$ and thus $\widetilde{\omega}$ is a weight function. 
For calculating the weight coefficients in (2.7) we can use the numerically stable methods from [16], 28, [43].

2.2. Explicit expressions of the generalized Stieltjes polynomials. We now study some cases of the quadrature formula (2.7) in detail for a subclass of the Gori-Micchelli weight functions, as well as for the generalized Chebyshev weights.

- First, consider a subclass of the Gori-Micchelli weight functions,

$$
\omega_{n, \ell}(t)=\left[\frac{U_{n-1}(t)}{n}\right]^{2 \ell}\left(1-t^{2}\right)^{\ell-1 / 2}, \quad \ell \in\{0,1, \ldots, s\}, \quad s \in \mathbb{N} .
$$

In the particular case $\ell=0$, (2.15) reduces to the Chebyshev weight function of first kind $\omega_{n, 0}(t)=\left(1-t^{2}\right)^{-1 / 2}$.

Recall that for the weight functions (2.15), the Chebyshev polynomials of first kind $T_{n}$ are $s$-orthogonal. In this case $\left(\sigma=\sigma_{n}=(s, s, \ldots, s)\right)$, the orthogonality conditions (2.9) have the form

$$
\int_{-1}^{1}\left[T_{n}(t)\right]^{2 s+1} \prod_{\mu=1}^{n+1}\left(t-x_{\mu}^{*}\right)^{2 s_{\mu}^{*}+1} t^{k} U_{n-1}^{2 \ell}(t)\left(1-t^{2}\right)^{\ell-1 / 2} d t=0,
$$

where $k=0,1, \ldots, n$. If

$$
\alpha \prod_{\mu=1}^{n+1}\left(t-x_{\mu}^{*}\right)^{2 s_{\mu}^{*}+1}=\left[U_{n-1}(t)\right]^{2 s+1-2 \ell}\left(t^{2}-1\right)^{s+1-\ell},
$$

where $\alpha$ is a normalization constant, then the conditions (2.16) obtain the form

$$
\int_{-1}^{1}\left[U_{n-1}(t) T_{n}(t)\right]^{2 s+1} t^{k}\left(1-t^{2}\right)^{1 / 2+s} d t=0, \quad k=0,1, \ldots, n,
$$

and since $2 T_{n}(t) U_{n-1}(t)=U_{2 n-1}(t)$ (cf. Monegato [32, Eq. (21), p. 143]),

$$
\int_{-1}^{1}\left[U_{2 n-1}(t)\right]^{2 s+1} t^{k}\left(1-t^{2}\right)^{1 / 2+s} d t=0, \quad k=0,1, \ldots, n .
$$

In fact, the last conditions hold for $k=0,1, \ldots, 2 n-2$ (see 34), which means that it has to be $2 n-2 \geq n$, i.e., $n \geq 2$. Therefore, in this case $\left(n \geq 2, \sigma_{n+1}^{*}=\right.$ $((s-\ell) / 2, s-\ell, \ldots, s-\ell,(s-\ell) / 2))$, when the quadrature formula $(2.2)$ has the form $\left(x_{1}^{*}=-1, x_{n+1}^{*}=1\right)$,

$$
\begin{aligned}
\int_{-1}^{1} f(t) \omega_{n, \ell}(t) d t \approx & \sum_{\nu=1}^{n} \sum_{i=0}^{2 s} b_{\nu i} f^{(i)}\left(x_{\nu}\right)+\sum_{\mu=2}^{n} \sum_{j=0}^{2(s-\ell)} c_{\mu j}^{*} f^{(j)}\left(x_{\mu}^{*}\right) \\
& +\sum_{j=0}^{s-\ell}\left(c_{1, j}^{*} f^{(j)}(-1)+c_{n+1, j}^{*} f^{(j)}(1)\right),
\end{aligned}
$$

we have just proved the following statement.

Theorem 2.5. In the Kronrod extension (2.17) of the Gauss-Turán quadrature formula (2.5), where $s_{\nu}=s, \nu=1, \ldots, n$, with the weight function (2.15), and for $n \geq 2$, the corresponding generalized Stieltjes polynomial $E_{n+1}^{\left(\sigma^{*}\right)}(t)$ is given by $E_{n+1}^{\left(\sigma^{*}\right)}(t) \equiv\left(t^{2}-1\right) U_{n-1}(t)$; i.e., the nodes $x_{\mu}^{*}, \mu=2, \ldots, n$, are the zeros of the Chebyshev polynomial of second kind $U_{n-1}(t)$ and $x_{1}^{*}=-1, x_{n+1}^{*}=1$. 
The zeros of $T_{n}(t)$ and $E_{n+1}^{\left(\sigma^{*}\right)}(t)$ interlace (i.e., satisfy the property (2.8)), since $2\left(t^{2}-1\right) U_{n-1}(t)=2 n^{-1}\left(t^{2}-1\right) T_{n}^{\prime}(t)$ (cf. [41, Lemma 1, p. 180]).

The algebraic degree of precision of the quadrature formula (2.17) as a Kronrod extension is $N=n(4 s-2 \ell+3)+1$.

Corollary 2.6. There exists a unique quadrature formula of the form

$$
\int_{-1}^{1} f(t)\left(1-t^{2}\right)^{1 / 2+s}\left[U_{n-1}(t)\right]^{2 s+1} d t \approx \sum_{\nu=1}^{n} \sum_{i=0}^{2 s} \widetilde{b}_{\nu i} f^{(i)}\left(x_{\nu}\right)
$$

of $A D P=2 n(s+1)(n \geq 2)$. Its nodes $x_{\nu}$ are the zeros of the Chebyshev orthogonal polynomial of first kind $T_{n}$ and the coefficients can be calculated using (2.4) via the coefficients of quadrature formula (2.17).

There also exists a unique quadrature formula of the form

$$
\begin{aligned}
\int_{-1}^{1} f(t) \omega_{n, \ell}(t)\left[T_{n}(t)\right]^{2 s+1} d t \approx & \sum_{\mu=2}^{n} \sum_{j=0}^{2(s-\ell)} \widetilde{c}_{\mu j}^{*} f^{(j)}\left(x_{\mu}^{*}\right) \\
& +\sum_{j=0}^{s-\ell}\left[\widetilde{c}_{1, j}^{*} f^{(j)}(-1)+\widetilde{c}_{n+1, j}^{*} f^{(j)}(1)\right]
\end{aligned}
$$

of $A D P=2 n(s-\ell+1)+1(n \geq 2)$. Its nodes $x_{\mu}^{*}$ are the zeros of the Chebyshev orthogonal polynomial of second kind $U_{n-1}(t)$ and the coefficients can be calculated using (2.4) via the coefficients of quadrature formula (2.17).

We see that the first interpolatory quadrature formula in this corollary is the quadrature formula (2.21) below with $n-1$ nodes. Thus, it has the algebraic degree of precision $(n-1)(2 s+3)+2 s+1$, and we conclude that the quadrature formula (2.17) has the algebraic degree of precision $\mathrm{ADP}=N=2 n(2 s-\ell+2)-1(n \geq 2)$. Therefore, the quadrature formula (2.17) is the Chakalov-Popoviciu-Lobatto type quadrature formula. Finally, the second quadrature formula in Corollary 2.6 has the algebraic degree of precision $\mathrm{ADP}=n(2 s-2 \ell+3)-1$, and it is the Gaussian quadrature formula in the cases when $s-\ell$ is odd.

Consider now the generalized Chebyshev weight functions of second, third and fourth kind.

- Let $\sigma_{n}=(s, \ldots, s)$ and $\omega(t) \equiv w_{2}(t)=\left(1-t^{2}\right)^{1 / 2+s}$. The orthogonality conditions (2.9), in this case, reduce to

$$
\int_{-1}^{1}\left[U_{n}(t)\right]^{2 s+1} \prod_{\mu=1}^{n+1}\left(t-x_{\mu}^{*}\right)^{2 s_{\mu}^{*}+1} t^{k}\left(1-t^{2}\right)^{1 / 2+s} d t=0, \quad k=0,1, \ldots, n
$$

If $\beta \prod_{\mu=1}^{n+1}\left(t-x_{\mu}^{*}\right)^{2 s_{\mu}^{*}+1}=T_{n+1}^{2 s+1}(t)$, where $\beta$ is a normalization constant and $\sigma_{n+1}^{*}=(s, s, \ldots, s)$, then the conditions (2.18) obtain the form, since $T_{n+1}(t) U_{n}(t)=U_{2 n+1}(t) / 2$,

$$
\int_{-1}^{1}\left[U_{2 n+1}(t)\right]^{2 s+1} t^{k}\left(1-t^{2}\right)^{1 / 2+s} d t=0, \quad k=0,1, \ldots, n .
$$


In fact, the last conditions hold for $k=0,1, \ldots, 2 n$. Therefore, in this case, when the quadrature formula (2.7) has the form

$$
\int_{-1}^{1} f(t)\left(1-t^{2}\right)^{1 / 2+s} d t \approx \sum_{\nu=1}^{n} \sum_{i=0}^{2 s} b_{\nu i} f^{(i)}\left(x_{\nu}\right)+\sum_{\mu=1}^{n+1} \sum_{j=0}^{2 s} c_{\mu j}^{*} f^{(j)}\left(x_{\mu}^{*}\right),
$$

we have just proved the following statement:

Theorem 2.7. In the Kronrod extension (2.19) of the Gauss-Turán quadrature formula (2.5), where $s_{\nu}=s, \nu=1, \ldots, n$, with the weight function $\omega_{2}(t)=$ $\left(1-t^{2}\right)^{1 / 2+s}$, the corresponding generalized Stieltjes polynomial $E_{n+1}^{\left(\sigma^{*}\right)}(t)\left(\sigma_{n+1}^{*}=\right.$ $(s, s, \ldots, s))$ is given by $E_{n+1}^{\left(\sigma^{*}\right)}(t) \equiv T_{n+1}(t)$; i.e., the nodes $x_{\mu}^{*}, \mu=1, \ldots, n+1$, are the zeros of the Chebyshev polynomial $T_{n+1}(t)$.

It is obvious that in this case the interlacing property (2.8) holds, since it holds for the polynomials $U_{n}(t)$ and $T_{n+1}(t)$. The algebraic degree of precision of the quadrature formula (2.19) is $N=2(2 n+1)(s+1)$, since it is in fact the GaussTurán quadrature.

Corollary 2.8. There exists a unique Gaussian quadrature formula of the form

$$
\int_{-1}^{1} f(t)\left(1-t^{2}\right)^{1 / 2+s}\left[T_{n+1}(t)\right]^{2 s+1} d t \approx \sum_{\nu=1}^{n} \sum_{i=0}^{2 s} \widetilde{b}_{\nu i} f^{(i)}\left(x_{\nu}\right),
$$

of $A D P=n(2 s+3)$. Its nodes $x_{\nu}$ are the zeros of the Chebyshev polynomial of second kind $U_{n}$ and the coefficients can be calculated using (2.4) via the coefficients of quadrature formula (2.19).

There also exists a unique Gaussian quadrature formula of the form

$$
\int_{-1}^{1} f(t)\left(1-t^{2}\right)^{1 / 2+s}\left[U_{n}(t)\right]^{2 s+1} d t \approx \sum_{\mu=1}^{n+1} \sum_{j=0}^{2 s} \widetilde{c}_{\mu j}^{*} f^{(j)}\left(x_{\mu}^{*}\right)
$$

of $A D P=n(2 s+3)+2 s+1$. Its nodes $x_{\mu}^{*}$ are the zeros of the Chebyshev polynomial of first kind $T_{n+1}$ and the coefficients can be calculated using (2.4) via the coefficients of quadrature formula (2.19).

- Let $\sigma_{n}=(s, \ldots, s)$ and $\omega(t) \equiv \omega_{4}(t)=(1-t)^{1 / 2+s}(1+t)^{-1 / 2}$. The orthogonality conditions (2.9), in this case, reduce to

$$
\begin{array}{r}
\int_{-1}^{1}\left[P_{n}^{(1 / 2,-1 / 2)}(t)\right]^{2 s+1} \prod_{\mu=1}^{n+1}\left(t-x_{\mu}^{*}\right)^{2 s_{\mu}^{*}+1} t^{k}(1-t)^{1 / 2+s}(1+t)^{-1 / 2} d t=0 \\
k=0,1, \ldots, n,
\end{array}
$$

where $P_{n}^{(1 / 2,-1 / 2)}$ is the Jacobi polynomial orthogonal on $(-1,1)$ with respect to the weight function $(1-t)^{1 / 2}(1+t)^{-1 / 2}$ (see [34]). If

$$
\gamma \prod_{\mu=1}^{n+1}\left(t-x_{\mu}^{*}\right)^{2 s_{\mu}^{*}+1}=(1+t)^{s+1}\left[P_{n}^{(-1 / 2,1 / 2)}(t)\right]^{2 s+1},
$$


where $\gamma$ is a normalization constant and $\sigma_{n+1}^{*}=(s / 2, s, \ldots, s)$, then the conditions (2.22) reduce to the form

$$
\int_{-1}^{1}\left[U_{2 n}(t)\right]^{2 s+1} t^{k}\left(1-t^{2}\right)^{1 / 2+s} d t=0, \quad k=0,1, \ldots, n,
$$

since $P_{n}^{(1 / 2,-1 / 2)}(t) P_{n}^{(-1 / 2,1 / 2)}(t)=$ const $\cdot U_{2 n}(t)$ (cf. [32, Equation (33), p. 147]). In fact, these conditions hold for $k=0,1, \ldots, 2 n-1$. Therefore, in this case, when the quadrature formula (2.7) has the form $\left(x_{1}^{*}=-1\right)$

$$
\int_{-1}^{1} f(t) \omega_{4}(t) d t \approx \sum_{\nu=1}^{n} \sum_{i=0}^{2 s} b_{\nu i} f^{(i)}\left(x_{\nu}\right)+\sum_{\mu=2}^{n+1} \sum_{j=0}^{2 s} c_{\mu j}^{*} f^{(j)}\left(x_{\mu}^{*}\right)+\sum_{j=0}^{s} c_{1, j}^{*} f^{(j)}(-1),
$$

we have just proved the following statement:

Theorem 2.9. In the Kronrod extension (2.23) of the Gauss-Turán quadrature formula (2.5), where $s_{\nu}=s, \nu=1, \ldots, n$, with the weight function $\omega_{4}(t)=$ $(1-t)^{1 / 2+s}(1+t)^{-1 / 2}$ the corresponding generalized Stieltjes polynomial $E_{n+1}^{\left(\sigma^{*}\right)}(t)$ $\left(\sigma_{n+1}^{*}=(s / 2, s, \ldots, s)\right)$ is given by $E_{n+1}^{\left(\sigma^{*}\right)}(t) \equiv(t+1) P_{n}^{(-1 / 2,1 / 2)}(t)$; i.e., the nodes $x_{\mu}^{*}, \mu=2, \ldots, n+1$, are the zeros of $P_{n}^{(-1 / 2,1 / 2)}$ and $x_{1}^{*}=-1$.

It is obvious that for this case the interlacing property (2.8) holds, since it holds for the zeros of the polynomials $P_{n}^{(1 / 2,-1 / 2)}$ and $(t+1) P_{n}^{(-1 / 2,1 / 2)}(t)$.

The algebraic degree of precision of the quadrature formula (2.23) as a Kronrod extension is $N=n(4 s+3)+s+1$.

Corollary 2.10. There exists a unique quadrature formula of the form

$$
\int_{-1}^{1} f(t)\left(1-t^{2}\right)^{1 / 2+s}\left[P_{n}^{(-1 / 2,1 / 2)}(t)\right]^{2 s+1} d t \approx \sum_{\nu=1}^{n} \sum_{i=0}^{2 s} \widetilde{b}_{\nu i} f^{(i)}\left(x_{\nu}\right)
$$

of $A D P=n(2 s+3)-1$. Its nodes $x_{\nu}$ are the zeros of the Chebyshev orthogonal polynomial of fourth kind $P_{n}^{(1 / 2,-1 / 2)}$ and the coefficients can be calculated using (2.4) via the coefficients of quadrature formula (2.23).

There also exists a unique quadrature formula of the form

$$
\int_{-1}^{1} f(t) \frac{(1-t)^{1 / 2+s}}{(1+t)^{1 / 2}}\left[P_{n}^{(1 / 2,-1 / 2)}(t)\right]^{2 s+1} d t \approx \sum_{\mu=2}^{n+1} \sum_{j=0}^{2 s} \widetilde{c}_{\mu j}^{*} f^{(j)}\left(x_{\mu}^{*}\right)+\sum_{j=0}^{s} \widetilde{c}_{1, j}^{*} f^{(j)}(-1)
$$

of $A D P=n(2 s+3)+s$. Its nodes $x_{\mu}^{*}$ are the zeros of the Chebyshev orthogonal polynomial of third kind $P_{n}^{(-1 / 2,1 / 2)}$ and the coefficients can be calculated using (2.4) via the coefficients of quadrature formula (2.23).

Proof. Consider the quadrature formula (2.23). By using Theorem 2.2 we conclude that the corresponding quadrature formula

$$
\int_{-1}^{1} f(t)\left(1-t^{2}\right)^{1 / 2+s} d t \approx \sum_{\nu=1}^{n} \sum_{i=0}^{2 s} \hat{b}_{\nu i} f^{(i)}\left(x_{\nu}\right)+\sum_{\mu=2}^{n+1} \sum_{j=0}^{2 s} \hat{c}_{\mu j}^{*} f^{(j)}\left(x_{\mu}^{*}\right)
$$

has the algebraic degree of precision $N=4 n(s+1)-1$, and the quadrature formula (2.23) as the Gauss-Turán-Radau type quadrature has the algebraic degree of precision $4 n(s+1)+s$. The assertion of the corollary follows. 
Both quadrature formulas from Corollary 2.10 are the Gaussian ones in the cases when $s$ is odd.

- When the quadrature formula (2.7) has the form $\left(x_{n+1}^{*}=1\right)$,

$$
\int_{-1}^{1} f(t) \omega_{3}(t) d t \approx \sum_{\nu=1}^{n} \sum_{i=0}^{2 s} b_{\nu i} f^{(i)}\left(x_{\nu}\right)+\sum_{\mu=1}^{n} \sum_{j=0}^{2 s} c_{\mu j}^{*} f^{(j)}\left(x_{\mu}^{*}\right)+\sum_{j=0}^{s} c_{n+1, j}^{*} f^{(j)}(1),
$$

where $\omega_{3}(t)=(1-t)^{-1 / 2}(1+t)^{1 / 2+s}$ is the generalized Chebyshev weight of third kind, in a similar way as in the previous case, the following statement can be proved.

Theorem 2.11. In the previous Kronrod extension of the Gauss-Turán quadrature formula (2.5), where $s_{\nu}=s, \nu=1, \ldots, n$, with the weight function $\omega_{3}(t)=$ $(1-t)^{-1 / 2}(1+t)^{1 / 2+s}$, the corresponding generalized Stieltjes polynomial $E_{n+1}^{\left(\sigma^{*}\right)}(t)$ $\left(\sigma_{n+1}^{*}=(s, \ldots, s, s / 2)\right)$ is given by $E_{n+1}^{\left(\sigma^{*}\right)}(t) \equiv(t-1) P_{n}^{(1 / 2,-1 / 2)}(t)$; i.e., the nodes $x_{\mu}^{*}, \mu=1, \ldots, n$, are the zeros of $P_{n}^{(1 / 2,-1 / 2)}(t)$ and $\tau_{n+1}^{*}=1$.

Now, an analogous corollary as Corollary 2.10 can be formulated.

We finish this section by adding some remarks concerning the computational aspects of the results by Bojanov and Petrova [3], and by extending their results from [3. Corollary 2.5] to the more general case.

Corollary 2.3 in 3 says that if one chooses points $x_{1}<x_{2}<\cdots<x_{n}$ and even multiplicities $\nu_{j}, j=1, \ldots, n$, so that $\Lambda(t ; \mathbf{x})$ is the corresponding $\sigma$-orthogonal $\left(\sigma=\left(\nu_{1} / 2, \ldots, \nu_{n} / 2\right)\right)$ polynomial with respect to the weight $\omega$, then it is possible to construct the monic polynomial $P_{k}(t)=\left(t-y_{1}\right) \cdots\left(t-y_{k}\right)$ orthogonal on $[a, b]$ with respect to the measure $\omega(t) \Lambda^{\bar{\nu}}(t ; \mathbf{x})$ (see [10], [12], [18]). Therefore, the points $y_{m}$, $m=1, \ldots, k$, with multiplicity 1 , and the points $x_{j}$ with multiplicities $\nu_{j}+1, j=$ $1, \ldots, n$, are the nodes in the uniquely determined Gaussian quadrature formula,

$$
\int_{a}^{b} \omega(t) f(t) d t \approx \sum_{m=1}^{k} b_{m 0} f\left(y_{m}\right)+\sum_{j=1}^{n} \sum_{i=0}^{\nu_{j}-1} a_{j i} f^{(i)}\left(x_{j}\right),
$$

with $\mathrm{ADP}=2 k+\sum_{j=1}^{n} \nu_{j}+n-1$. The coefficients of the last quadrature are calculated as the ones in the interpolatory quadrature formula (see [28], [43]). Now, the coefficients $c_{j i}\left(j=1, \ldots, n ; i=0,1, \ldots, \nu_{j}-1\right)$ in the corresponding quadrature formula (1.3) are calculated by (2.4), where $a_{j i}\left(j=1, \ldots, n ; i=0,1, \ldots, \nu_{j}-1\right)$ are from (2.24).

Corollary 2.12. For any given set of even multiplicities $\bar{\nu}=\left(\nu_{1}, \ldots, \nu_{n}\right)$ and weight $\omega$, there exists a unique Gaussian quadrature (with $A D P=\nu_{1}+\cdots+\nu_{n}+n-1$ ) of the form

$$
\int_{a}^{b} \omega(t) \Lambda(t ; \mathbf{x}) f(t) d t \approx \sum_{j=1}^{n} \sum_{i=0}^{\nu_{j}-1} c_{j i} f^{(i)}\left(x_{j}\right) .
$$

Its nodes $\left\{x_{j}\right\}_{j=1}^{n}$ are the nodes of the Chakalov-Popoviciu quadrature formula

$$
\int_{a}^{b} \omega(t) f(t) d t \approx \sum_{j=1}^{n} \sum_{i=0}^{\nu_{j}} a_{j i} f^{(i)}\left(x_{j}\right)
$$

of $A D P=\nu_{1}+\cdots+\nu_{n}+2 n-1$, and $\operatorname{sign}\left(c_{j, \nu_{j}-1}\right)=(-1)^{n-j}, j=1, \ldots, n$. 
Proof. Since the first part of this corollary is stated the same as Corollary 2.4 in [3], it only remains to prove the last part. Applying (2.26) to $\Lambda(\cdot ; \mathbf{x}) Q$, where $Q$ is a polynomial of degree $\nu_{1}+\cdots+\nu_{n}+n-1$, we have that

$$
\begin{array}{r}
{[\Lambda(t ; \mathbf{x}) Q(t)]^{(i)}\left(x_{j}\right)=i \Lambda_{j}\left(x_{j} ; \mathbf{x}\right) Q^{(i-1)}\left(x_{j}\right)+\sum_{\ell=0}^{i-2} d_{\ell j} Q^{(\ell)}\left(x_{j}\right),} \\
i=2, \ldots, \min \left(n, \nu_{j}\right),
\end{array}
$$

and therefore we obtain (2.25), where

$$
c_{j i}=\sum_{s=i+1}^{\nu_{j}} a_{j s}\left(\begin{array}{l}
s \\
i
\end{array}\right) \Lambda^{(s-i)}\left(x_{j} ; \mathbf{x}\right) \quad\left(j=1, \ldots, n ; i=0,1, \ldots, \nu_{j}-1\right) .
$$

Therefore, $c_{j, \nu_{j}-1}=a_{j, \nu_{j}}\left(\begin{array}{c}\nu_{j} \\ \nu_{j}-1\end{array}\right) \Lambda^{\prime}\left(x_{j} ; \mathbf{x}\right)=\nu_{j} a_{j, \nu_{j}} \Lambda_{j}\left(x_{j} ; \mathbf{x}\right)$, and since $a_{j, \nu_{j}}>0$, when $\nu_{j}$ is even, we conclude that $\operatorname{sign}\left(c_{j, \nu_{j}-1}\right)=\operatorname{sign}\left(\Lambda_{j}\left(x_{j} ; \mathbf{x}\right)\right)=(-1)^{n-j}, j=$ $1, \ldots, n$. The proof follows.

\section{Connection to Gauss-Kronrod And generalized AVERAGed GAUSSIAN QUADRATURE FORMULAS}

Consider quadrature formulas of the type

$$
\int_{a}^{b} f(t) P_{n}(t) \omega(t) d t \approx \sum_{j=1}^{n+1} b_{j} f\left(x_{j}\right), \quad P_{n}(t)=\left(t-y_{1}\right)\left(t-y_{2}\right) \cdots\left(t-y_{n}\right),
$$

where $\mathbf{y}:=\left(y_{1}, \ldots, y_{n}\right)$ is a given set of points and $y_{i} \neq x_{j}$ for all $i$ and $j$. Let $P_{n}(t)$ be the monic orthogonal polynomial with respect to the weight function $\omega(t)$.

Following Bojanov and Petrova [3, Sections 3.1, 3.2]), we associate with (3.1) the quadrature formula

$$
\int_{a}^{b} f(t) \omega(t) d t \approx \sum_{j=1}^{n} \alpha_{j} f\left(y_{j}\right)+\sum_{j=1}^{n+1} \beta_{j} f\left(x_{j}\right) .
$$

We know that (3.2) has ADP $=N$ if and only if the quadrature (3.1) has ADP $=$ $N-n$. The coefficients $b_{j}, j=1, \ldots, n+1$, are given by the relation (see [3, Section $3.2]$ )

$$
b_{j}=\beta_{j} P_{n}\left(x_{j}\right), \quad j=1, \ldots, n+1 .
$$

Lemma 3.4 in [3] shows that the question of constructing formulas of type (3.1) of the highest ADP with $n+1$ nodes in a special case when $P_{n}(t)$ is fixed to be the $n$-th polynomial on $[a, b]$ with the weight $\omega$ becomes a question of constructing GaussKronrod formulas of type (3.2) on $[a, b]$ with the weight $\omega$. In fact, in this case, $N=3 n+1$, and $\operatorname{ADP}(3.1)=N-n=2 n+1$. In the case when the corresponding Gauss-Kronrod quadrature formula is positive, there exist the effective numerical procedures of its numerically stable construction given by Laurie [23] and Calvetti et al. [4] (see also Monegato [33]).

The existence of the positive Gauss-Kronrod q.f. depends on $\omega$, and there are several cases of non-existence known, e.g., for the Gauss-Laguerre and GaussHermite cases [19. Recently, for the Gegenbauer weight $\omega^{(\alpha, \alpha)}(t)=\left(1-t^{2}\right)^{\alpha}$, Peherstorfer and Petras [36] have shown nonexistence of Gauss-Kronrod formulas for $n$ sufficiently large and $\alpha>5 / 2$. Analogous results for the Jacobi weight 
function $\omega^{(\alpha, \beta)}(t)=(1-t)^{\alpha}(1+t)^{\beta}$ can be found in their paper [37], particularly, nonexistence for large $n$ of Gauss-Kronrod formulas when $\min (\alpha, \beta) \geq 0$ and $\max (\alpha, \beta)>5 / 2$. In such cases it is of interest to find an adequate alternative to the corresponding Gauss-Kronrod quadrature formulae.

An alternative approach are the Anti-Gaussian formulas introduced by Laurie [22], which have been slightly generalized in [6], and in Spalević's paper [39. Such formulas always exist and are positive. In 39] a very simple numerical method is proposed for constructing the averaged Gaussian quadrature formulas. In [40] Spalevic tried to answer whether the averaged Gaussian formulas are an adequate alternative to the corresponding Gauss-Kronrod quadrature formulas to estimate the remainder term of a Gaussian rule.

The maximal polynomial degree of precision of the generalized averaged Gaussian quadrature formulas (3.1) in general is $N=2 n+2(2 n+3$ in the symmetric case $[a, b]=[-c, c], \omega(-t)=\omega(t)$ ) (see [39, p. 1486]). Therefore, in these cases $\operatorname{ADP}(3.1)=N-n=n+2(n+3$ in the symmetric case $)$.

Example. In [39, Ex. 2.2] the Jacobi weight function $\omega^{(\alpha, \beta)}(t)$ with $\alpha=1 / 10, \beta=$ $13 / 5$, on $[-1,1]$ is considered. Respective Matlab routines $r_{-} j a c o b i . m$, for the coefficients in the three-term recurrence relation of the corresponding Jacobi orthogonal polynomials, and gauss.m, for the nodes and weight coefficients in the corresponding Gauss quadrature formula, are downloadable from the website of Walter Gautschi; it contains a suite of many other useful routines, in part assembled as a companion piece to the book 10. So, the nodes and corresponding weight coefficients of the corresponding quadrature formula (3.2) are displayed in the table in [39, p. 1488].

As we have observed there, the corresponding quadrature formula (3.2), which has the degree of exactness $2 n+2$, is an extension of the Gauss formula. Nonexistence for large $n$ of Gauss-Kronrod formulae, for the case of Jacobi weight functions under consideration has been proved by Peherstorfer and Petras 35. Using the Matlab routine kronrod.m, which is downloadable from the above-mentioned website, we obtained that the Gauss-Kronrod quadrature formula does not exist in the considered case $\left(\omega^{(1 / 10,13 / 5)}(t), n=14\right)$. For $1 \leq n \leq 13$ the Matlab routine kronrod.m generates the corresponding Gauss-Kronrod quadrature formulas.

Now, the nodes and the weight coefficients in (3.1) in the considered Jacobi case, obtained by (3.3), are given as follows.

$$
\begin{aligned}
& x_{1}=-9.686625499734723 \mathrm{e}-001 \\
& x_{2}=-8.878728134056509 \mathrm{e}-001 \\
& x_{3}=-7.743502857984884 \mathrm{e}-001 \\
& x_{4}=-6.309958682484870 \mathrm{e}-001 \\
& x_{5}=-4.632684854851569 \mathrm{e}-001 \\
& x_{6}=-2.776956155761358 \mathrm{e}-001 \\
& x_{7}=-8.152901380092521 \mathrm{e}-002 \\
& x_{8}=1.175565290185397 \mathrm{e}-001 \\
& x_{9}=3.117686462151886 \mathrm{e}-001 \\
& x_{10}=4.935042803199599 \mathrm{e}-001 \\
& x_{11}=6.556481067408614 \mathrm{e}-001 \\
& x_{12}=7.918514028863188 \mathrm{e}-001 \\
& x_{13}=8.967804407043425 \mathrm{e}-001 \\
& x_{14}=9.663230344398555 \mathrm{e}-001 \\
& x_{15}=9.977311827889372 \mathrm{e}-001
\end{aligned}
$$

$$
\begin{array}{lr}
b_{1}= & 3.238722682811740 \mathrm{e}-008 \\
b_{2}= & -1.654833893907501 \mathrm{e}-007 \\
b_{3}= & 4.574035484062901 \mathrm{e}-007 \\
b_{4}= & -9.390505362302107 \mathrm{e}-007 \\
b_{5}= & 1.610843298436290 \mathrm{e}-006 \\
b_{6}= & -2.438855724849397 \mathrm{e}-006 \\
b_{7}= & 3.356667231984657 \mathrm{e}-006 \\
b_{8}= & -4.271741556540400 \mathrm{e}-006 \\
b_{9}= & 5.075337723677705 \mathrm{e}-006 \\
b_{10}= & -5.654368868938921 \mathrm{e}-006 \\
b_{11}= & 5.903012486495493 \mathrm{e}-006 \\
b_{12}= & -5.730895950696384 \mathrm{e}-006 \\
b_{13}= & 5.061534260973886 \mathrm{e}-006 \\
b_{14}= & -3.797258505019950 \mathrm{e}-006 \\
b_{15}= & 1.500468754863419 \mathrm{e}-006
\end{array}
$$




\section{Quadratures With FiXed multiple nodes}

Here, we discuss quadratures of the form

$$
\int_{a}^{b} \omega(t) \Lambda^{\bar{\mu}}(t ; \mathbf{y}) f(t) d t \approx \sum_{m=1}^{k} \sum_{s=0}^{\lambda_{m}-1} b_{m s} f^{(s)}\left(y_{m}\right)+\sum_{j=1}^{n} \sum_{i=0}^{\nu_{j}-1} a_{j i} f^{(i)}\left(x_{j}\right),
$$

where all $\left\{\mu_{i}\right\}_{i=1}^{k},\left\{\lambda_{m}\right\}_{m=1}^{k},\left\{\nu_{j}\right\}_{j=1}^{n}$ are odd nonnegative integers, which generalize the quadratures [3, Eq. (3.7)]. The nodes $y_{1}, \ldots, y_{k}$ are fixed and we look for $n$ other points $x_{1}, \ldots, x_{n}$, to obtain the highest possible ADP. Let us suppose in this section that $y_{i}, x_{j} \in(a, b)(i=1, \ldots, k ; j=1, \ldots, n)$, and $y_{i} \neq x_{j}$ for all $i, j$. Clearly, if $n=0$, the maximal ADP of such a formula will be $\lambda_{1}+\cdots+\lambda_{k}-1$, since the formula will not be exact for $\Lambda^{\bar{\mu}}(t ; \mathbf{y})$. In this case any interpolatory formula based on the nodes $\left\{y_{i}\right\}_{i=1}^{k}$ is Gaussian. The following theorem is true.

Theorem 4.1. For any given set of distinct points $\mathbf{y}=\left(y_{1}, \ldots, y_{k}\right)$ and odd multiplicities $\lambda_{1}, \ldots, \lambda_{k}$, there exists a unique quadrature formula of the form (4.1) of highest ADP (which is $\left.\sum_{m=1}^{k} \lambda_{m}+\sum_{j=1}^{n} \nu_{j}+n-1\right)$. The nodes $\left\{x_{j}\right\}_{j=1}^{n}$, with odd multiplicities $\bar{\nu}=\left(\nu_{1}, \ldots, \nu_{n}\right)$, coincide with the nodes of the Ghakalov-Popoviciu quadrature formula with $A D P=\sum_{j=1}^{n} \nu_{j}+n-1$,

$$
\int_{a}^{b} \widetilde{\omega}(t) g(t) d t \approx \sum_{j=1}^{n} \sum_{i=0}^{\nu_{j}-1} \alpha_{j i} g^{(i)}\left(x_{j}\right)
$$

where the weight function $\widetilde{\omega}$ is given by

$$
\widetilde{\omega}(t)=\omega(t) \Lambda^{\bar{\mu}}(t ; \mathbf{y}) \Lambda^{\bar{\lambda}}(t ; \mathbf{y})=\omega(t) \Lambda^{\bar{\mu}+\bar{\lambda}}(t ; \mathbf{y}),
$$

and the coefficients $a_{j i}$ in (4.1) are computed from the coefficients $\alpha_{j k}$ in (4.2) by

$$
a_{j i}=\sum_{k=i}^{\nu_{j}-1}\left(\begin{array}{l}
k \\
i
\end{array}\right)\left(\Lambda^{\bar{\lambda}}(t ; \mathbf{y})^{-1}\right)_{t=x_{j}}^{(k-i)} \alpha_{j k}, \quad j=1, \ldots, n ; i=0,1, \ldots, \nu_{j}-1 .
$$

Proof. Let $H$ be the Hermite interpolating polynomial of degree $\lambda_{1}+\cdots+\lambda_{k}-1$ for the function $f$ and nodes $\mathbf{y}$. Then, by Newton's formula,

$$
f(t)=H(t)+f\left[y_{1}^{\lambda_{1}}, \ldots, y_{k}^{\lambda_{k}}, t\right] \Lambda^{\bar{\lambda}}(t ; \mathbf{y}) .
$$

Multiplying this identity by $\omega(t) \Lambda^{\bar{\mu}}(t ; \mathbf{y})$ and integrating over $[a, b]$ we obtain

$$
\int_{a}^{b} \omega(t) \Lambda^{\bar{\mu}}(t ; \mathbf{y}) f(t) d t=\int_{a}^{b} \omega(t) \Lambda^{\bar{\mu}}(t ; \mathbf{y}) H(t) d t+\int_{a}^{b} \widetilde{\omega}(t) f\left[y_{1}^{\lambda_{1}}, \ldots, y_{k}^{\lambda_{k}}, t\right] d t
$$

where $\widetilde{\omega}$ is given by (4.3). It is well known that the Hermite interpolating polynomial of degree $\lambda_{1}+\cdots+\lambda_{k}-1$ has the form

$$
H(t)=\sum_{m=1}^{k} \sum_{s=0}^{\lambda_{m}-1} \ell_{m s}(t) f^{(s)}\left(y_{m}\right)
$$

where $\ell_{m s}(t)$ are the fundamental functions of Hermite interpolation, so that we have

$$
\int_{a}^{b} \omega(t) \Lambda^{\bar{\mu}}(t ; \mathbf{y}) H(t) d t=\sum_{m=1}^{k} \sum_{s=0}^{\lambda_{m}-1} C_{m s} f^{(s)}\left(y_{m}\right)
$$


where $C_{m s}=\int_{a}^{b} \omega(t) \Lambda^{\bar{\mu}}(t ; \mathbf{y}) \ell_{m s}(t) d t, m=1, \ldots, k ; s=0,1, \ldots, \lambda_{m}-1$. Furthermore, by using the uniqueness of the Chakalov-Popoviciu quadrature formula (4.2) with respect to the weight function $\widetilde{\omega}(t)$, given by (4.3), we have

$$
\int_{a}^{b} \widetilde{\omega}(t) f\left[y_{1}^{\lambda_{1}}, \ldots, y_{k}^{\lambda_{k}}, t\right] d t=\sum_{j=1}^{n} \sum_{i=0}^{\nu_{j}-1} \alpha_{j i} f^{(i)}\left[y_{1}^{\lambda_{1}}, \ldots, y_{k}^{\lambda_{k}}, x_{j}\right],
$$

which is exact for each polynomial $f$ of degree at most $\sum_{m=1}^{k} \lambda_{m}+\sum_{j=1}^{n} \nu_{j}+n-1$, since then, $f\left[y_{1}^{\lambda_{1}}, \ldots, y_{k}^{\lambda_{k}}, t\right]$ is a polynomial of degree $\sum_{j=1}^{n} \nu_{j}+n-1$. We have used the notation

$$
f^{(i)}\left[y_{1}^{\lambda_{1}}, \ldots, y_{k}^{\lambda_{k}}, x_{j}\right]=\left.\frac{d^{i}}{d t^{i}}\left(f\left[y_{1}^{\lambda_{1}}, \ldots, y_{k}^{\lambda_{k}}, t\right]\right)\right|_{t=x_{j}} .
$$

From (4.5), we have $f\left[y_{1}^{\lambda_{1}}, \ldots, y_{k}^{\lambda_{k}}, t\right]=f(t)\left[\Lambda^{\bar{\lambda}}(t ; \mathbf{y})\right]^{-1}-H(t)\left[\Lambda^{\bar{\lambda}}(t ; \mathbf{y})\right]^{-1}$ and

$$
\begin{aligned}
f^{(i)}\left[y_{1}^{\lambda_{1}}, \ldots, y_{k}^{\lambda_{k}}, t\right]= & \sum_{\nu=0}^{i}\left(\begin{array}{l}
i \\
\nu
\end{array}\right) f^{(\nu)}(t)\left[\Lambda^{\bar{\lambda}}(t ; \mathbf{y})^{-1}\right]^{(i-\nu)} \\
& -\sum_{\nu=0}^{i}\left(\begin{array}{l}
i \\
\nu
\end{array}\right) H^{(\nu)}(t)\left[\Lambda^{\bar{\lambda}}(t ; \mathbf{y})^{-1}\right]^{(i-\nu)} \\
= & \sum_{\nu=0}^{i}\left(\begin{array}{l}
i \\
\nu
\end{array}\right)\left[\Lambda^{\bar{\lambda}}(t ; \mathbf{y})^{-1}\right]^{(i-\nu)} \\
& \cdot\left\{f^{(\nu)}(t)-\sum_{m=1}^{k} \sum_{s=0}^{\lambda_{m}-1} \ell_{m s}^{(\nu)}(t) f^{(s)}\left(y_{m}\right)\right\} .
\end{aligned}
$$

By using the last equality, we obtain

$$
\begin{aligned}
\sum_{j=1}^{n} \sum_{i=0}^{\nu_{j}-1} \alpha_{j i} f^{(i)}\left[y_{1}^{\lambda_{1}}, \ldots, y_{k}^{\lambda_{k}}, x_{j}\right]= & \sum_{j=1}^{n} \sum_{i=0}^{\nu_{j}-1} \alpha_{j i} \sum_{\nu=0}^{i}\left(\begin{array}{l}
i \\
\nu
\end{array}\right)\left[\Lambda^{\bar{\lambda}}(t ; \mathbf{y})^{-1}\right]_{t=x_{j}}^{(i-\nu)} f^{(\nu)}\left(x_{j}\right) \\
& +\sum_{m=1}^{k} \sum_{s=0}^{\lambda_{m}-1} \widetilde{\alpha}_{m s} f^{(s)}\left(y_{m}\right),
\end{aligned}
$$

where

$$
\widetilde{\alpha}_{m s}=-\sum_{j=1}^{n} \sum_{i=0}^{\nu_{j}-1} \alpha_{j i} \sum_{\nu=0}^{i}\left(\begin{array}{l}
i \\
\nu
\end{array}\right)\left[\Lambda^{\bar{\lambda}}(t ; \mathbf{y})^{-1}\right]_{t=x_{j}}^{(i-\nu)} \ell_{m s}^{(\nu)}\left(x_{j}\right) .
$$

Finally, we can easily derive that

$$
\alpha_{j i} \sum_{\nu=0}^{i}\left(\begin{array}{l}
i \\
\nu
\end{array}\right)\left[\Lambda^{\bar{\lambda}}(t ; \mathbf{y})^{-1}\right]_{t=x_{j}}^{(i-\nu)} f^{(\nu)}\left(x_{j}\right)=a_{j i} f^{(i)}\left(x_{j}\right),
$$

where $a_{j i}\left(j=1, \ldots, n ; i=0,1, \ldots, \nu_{j}-1\right)$ are given by 4.4 .

Therefore, the coefficients $\left\{\alpha_{j i}\right\}\left(j=1, \ldots, n ; i=0,1, \ldots, \nu_{j}-1\right)$ and the nodes $\left\{x_{j}\right\}_{j=1}^{n}$ are uniquely characterized as parameters of the Gaussian quadrature (4.2). It follows that the coefficients $\left\{a_{j i}\right\}\left(j=1, \ldots, n ; i=0,1, \ldots, \nu_{j}-1\right)$ given by (4.4) and $\left\{b_{m s}\right\}\left(m=1, \ldots, k ; s=0,1, \ldots, \lambda_{m}-1\right)$ in (4.1) are uniquely determined because the formula (4.1) is of interpolatory type. The proof is completed. 
Theorem 4.1, which generalizes Theorem 3.6 in [3], shows that one can improve the precision of the quadrature

$$
\int_{a}^{b} \omega(t) \Lambda^{\bar{\mu}}(t ; \mathbf{y}) f(t) d t \approx \sum_{m=1}^{k} \sum_{s=0}^{\lambda_{m}-1} \beta_{m s} f^{(s)}\left(y_{m}\right), \quad \Lambda^{\bar{\mu}}(t ; \mathbf{y})=\prod_{i=1}^{k}\left(t-y_{i}\right)^{\mu_{i}}
$$

where $\mu_{i}(i=1, \ldots, k), \lambda_{m}(m=1, \ldots, k)$ are nonnegative odd integers, following the strategy of Kronrod, namely by adding additional nodes $\left\{x_{j}\right\}_{j=1}^{n}$ which are of multiplicities $\nu_{j}(j=1, \ldots, n)$, where $\nu_{j}(j=1, \ldots, n)$ are nonnegative odd integers. One can achieve the highest possible precision $\sum_{m=1}^{k} \lambda_{m}+\sum_{j=1}^{n} \nu_{j}+n-1$ only by adding specific nodes, the nodes of the Chakalov-Popoviciu quadrature formula (4.2) on $[a, b]$ with weight $\widetilde{\omega}$ given by (4.3).

Corollary 4.2. Let $\lambda_{1}=\cdots=\lambda_{k}=1$ and $P_{k}(t)=\Lambda(t ; \mathbf{y})=\left(t-y_{1}\right) \cdots\left(t-y_{k}\right)$ be the $k$-th $\sigma$-orthogonal polynomial with respect to the weight function $\omega$ on $[a, b]$, where $\sigma=\left(\left(\mu_{1}-1\right) / 2, \ldots,\left(\mu_{k}-1\right) / 2\right)$ and $\mu_{1}, \ldots, \mu_{k}$ are nonnegative odd integers. Then there exists a unique Gaussian quadrature (with $A D P=k+\sum_{j=1}^{n} \nu_{j}+n-1$ ) of type (4.1). This formula is

$$
\int_{a}^{b} \omega(t) \Lambda^{\bar{\mu}}(t ; \mathbf{y}) f(t) d t \approx \sum_{j=1}^{n} \sum_{i=0}^{\nu_{j}-1} \alpha_{j i} f^{(i)}\left[y_{1}, y_{2}, \ldots, y_{k}, x_{j}\right]
$$

where $\left\{\alpha_{j i}\right\}\left(j=1, \ldots, n ; i=0,1, \ldots, \nu_{j}-1\right)$ are the weights and $\left\{x_{j}\right\}_{j=1}^{n}$ are the nodes of the Chakalov-Popoviciu quadrature formula on $[a, b]$ with weight $\hat{\omega}(t)=$ $\omega(t) \Lambda^{\bar{\mu}}(t ; \mathbf{y}) \Lambda(t ; \mathbf{y})$,

$$
\int_{a}^{b} \hat{\omega}(t) g(t) d t \approx \sum_{j=1}^{n} \sum_{i=0}^{\nu_{j}-1} \alpha_{j i} g^{(i)}\left(x_{j}\right)
$$

Proof. Here, $H(t)=L_{k-1}(t)$, where $L_{k-1}(t)$ is the Lagrange interpolating polynomial of degree $k-1$ based on the nodes $y_{1}, \ldots, y_{k}$. The proof follows the argument in Theorem 4.1 and the fact that the first integral in (4.6), $\int_{a}^{b} \omega(t) \Lambda^{\bar{\mu}}(t ; \mathbf{y}) L_{k-1}(t) d t=$ 0 , because of the $\sigma$-orthogonality of $P_{k}$. This completes the proof.

A particularly interesting subcase of Corollary 4.2 is as follows:

Corollary 4.3. Let $\mu_{m}=\lambda_{m}=1, m=1, \ldots, k$, and let $P_{k}(t)=\Lambda(t ; \mathbf{y})=$ $\left(t-y_{1}\right) \cdots\left(t-y_{k}\right)$ be the $k$-th orthogonal polynomial with respect to the weight function $\omega$ on $[a, b]$. Then there exists a unique Gaussian quadrature (with ADP $\left.=k+\sum_{j=1}^{n} \nu_{j}+n-1\right)$ of type

$$
\int_{a}^{b} \omega(t) P_{k}(t) f(t) d t \approx \sum_{m=1}^{k} b_{m} f\left(y_{m}\right)+\sum_{j=1}^{n} \sum_{i=0}^{\nu_{j}-1} a_{j i} f^{(i)}\left(x_{j}\right) .
$$

This formula is

$$
\int_{a}^{b} \omega(t) P_{k}(t) f(t) d t \approx \sum_{j=1}^{n} \sum_{i=0}^{\nu_{j}-1} \alpha_{j i} f^{(i)}\left[y_{1}, y_{2}, \ldots, y_{k}, x_{j}\right],
$$

where $\left\{\alpha_{j i}\right\}\left(j=1, \ldots, n ; i=0,1, \ldots, \nu_{j}-1\right)$ are the weights and $\left\{x_{j}\right\}_{j=1}^{n}$ are the nodes of the Chakalov-Popoviciu quadrature formula (4.7), on $[a, b]$ with the weight function $\hat{\omega}(t)=\omega(t) P_{k}(t)^{2}$. 
A very popular method for obtaining a practical error estimate in numerical integration by standard quadratures is to use two quadrature formulae $A$ and $B$, where the nodes used by formula $B$ form a proper subset of those used by formula $A$, and where the rule $A$ is also of higher degree of precision. Kronrod originated this method (see [20]), which has been used many times to date. For more details concerning this theory for standard quadrature formulas, see, for example 32, 33, [22], 39], 40]. The difference $|A(f)-B(f)|$, where $f$ is the integrand, is usually quite a good estimate of the error for the rule $B$. We are not aware, in the theory of standard quadratures, of the case where $A$ is a quadrature based on Gauss nodes, and $B$ is its Kronrod extension with multiple nodes.

Let $P_{k}$ be the $k$-th orthogonal polynomial with respect to the weight $\omega$ on $[a, b]$. Theorem 3.6 of Bojanov and Petrova [3] shows that one can improve the precision of the quadrature formula $\int_{a}^{b} \omega(t) P_{k}(t) f(t) d t \approx \sum_{m=1}^{k} \beta_{m} f\left(y_{m}\right), P_{k}(t)=$ $\left(t-y_{1}\right) \cdots\left(t-y_{k}\right)$, following the strategy of Kronrod by adding additional nodes $\left\{x_{j}\right\}_{j=1}^{n}$. The case $n=k+1$ is best known. One can achieve the highest possible precision $3 k+1$ only by adding specific nodes, the nodes of the Gauss quadrature on $[a, b]$ with the weight $\omega P_{k}^{2}$. But, what we wish to point out here, on the basis of Corollary 4.3 is that one can achieve the highest possible precision $2 k+\sum_{j=1}^{k+1} \nu_{j}$ by adding additional nodes $\left\{x_{j}\right\}_{j=1}^{k+1}$ with odd multiplicities $\nu_{j}, j=1, \ldots, k+1$, being the nodes of the corresponding Chakalov-Popoviciu quadrature (where $n=k+1$ ) on $[a, b]$ with the weight $\omega P_{k}^{2}$.

Observe that the last quadrature formula is based on the nodes $y_{m}$, the zeros of the corresponding orthogonal polynomial $P_{k}$ with respect to the weight $\omega$. Its algebraic degree of precision is equal to $k-1$. Its coefficients in fact are equal to zero. Namely, by putting $f(t)=P_{k}(t) /\left(t-y_{m}\right)$, we have $0=$ $\int_{a}^{b} \omega(t) P_{k}(t) P_{k}(t) /\left(t-y_{m}\right) d t=\beta_{m} P_{k}^{\prime}\left(y_{m}\right)$, which implies $\beta_{m}=0, m=1, \ldots, k$.

Let $\hat{T}_{n}$ and $\hat{U}_{n}$ be the monic Chebyshev polynomials of the first and second kind, respectively. For the subclass of Gori-Micchelli weight functions of the type (2.15), $\hat{\omega}_{n, s}(t)=\left(1-t^{2}\right)^{1 / 2+s}\left[\hat{U}_{n}(t)\right]^{2 s+2}, s \in \mathbb{N}_{0}$, the coefficients $\left\{\alpha_{j i}\right\}(j=1, \ldots, n+$ $1 ; i=0,1, \ldots, 2 p)$ of the Gauss-Turán quadrature formulas

$$
\int_{-1}^{1}\left(1-t^{2}\right)^{1 / 2+s}\left[\hat{U}_{n}(t)\right]^{2 s+2} f(t) d t \approx \sum_{j=1}^{n+1} \sum_{i=0}^{2 p} \alpha_{j i} f^{(i)}\left(\xi_{j}\right), \quad p \in \mathbb{N},
$$

can be explicitly computed (cf. Gori and Micchelli [17, and especially Yang [45]). The nodes $\xi_{j}, j=1, \ldots, n+1$, are the zeros of $\hat{T}_{n+1}$. From Corollary 4.2 the following corollary immediately follows.

Corollary 4.4. The quadrature formula

$$
\int_{-1}^{1}\left(1-t^{2}\right)^{1 / 2+s}\left[\hat{U}_{n}(t)\right]^{2 s+1} f(t) d t \approx \sum_{j=1}^{n+1} \sum_{i=0}^{2 p} \alpha_{j i} f^{(i)}\left[\eta_{1}, \eta_{2}, \ldots, \eta_{n}, \xi_{j}\right],
$$

where $s \in \mathbb{N}_{0}, p \in \mathbb{N}$, and $\alpha_{j i}, \xi_{j}$ are the parameters which appear in (4.9), and $\eta_{1}, \ldots, \eta_{n}$ are the zeros of $\hat{U}_{n}$, is the only Gaussian quadrature formula (exact for all polynomials of degree $(2 p+3) n+2 p+1)$, of the form (4.1) with $\omega(t)=\left(1-t^{2}\right)^{1 / 2+s}$, $k:=n, n:=n+1, \mu_{i}=2 s+1, \lambda_{i}=1, i=1, \ldots, n ; \nu_{j}=2 p+1, j=1, \ldots, n+1$; $\Lambda^{\bar{\mu}}(t ; \mathbf{y})=\left[\hat{U}_{n}(t)\right]^{2 s+1}$. 
We end this section by finding an example that confirms the interesting and important statement of Corollary 4.3, which is an immediate consequence of Corollary 4.4 for $s=0$. Namely, we can construct the Kronrod extension, with multiple nodes and highest ADP, of the quadrature formula for Chebyshev-Fourier coefficients of the second kind, which is based on the zeros of $\hat{U}_{n}$, and with $\beta_{m}=0, m=1, \ldots, n$,

$$
\int_{-1}^{1} \sqrt{1-t^{2}} \hat{U}_{n}(t) f(t) d t \approx \sum_{m=1}^{n} \beta_{m} f\left(\eta_{m}\right)
$$

as follows.

Corollary 4.5. The quadrature formula

$$
\int_{-1}^{1} \sqrt{1-t^{2}} \hat{U}_{n}(t) f(t) d t \approx \sum_{j=1}^{n+1} \sum_{i=0}^{2 p} \alpha_{j i} f^{(i)}\left[\eta_{1}, \ldots, \eta_{n}, \xi_{j}\right]
$$

where $p \in \mathbb{N}$, and $\alpha_{j i}, \xi_{j}$ are the parameters which appear in (4.9) with $s=0$, and $\eta_{1}, \ldots, \eta_{n}$ are the zeros of $\hat{U}_{n}$, is the only Gaussian quadrature formula (exact for all polynomials of degree $(2 p+3) n+2 p+1)$, of the form (4.8) with $\omega(t)=\sqrt{1-t^{2}}$, $k:=n, n:=n+1, \mu_{i}=\lambda_{i}=1, i=1, \ldots, n ; \nu_{j}=2 p+1, j=1, \ldots, n+1$; $P_{n}=\hat{U}_{n}$.

Example. The nodes in (4.9) are zeros of $\hat{T}_{n+1}$ given by $\xi_{j}=\cos \frac{(2 j-1) \pi}{2(n+1)}, j=$ $1, \ldots, n+1$. The coefficients $\alpha_{j i}$ are given by (see [45, Eq. (3.5)])

$$
\begin{gathered}
\alpha_{j 0}=\frac{\pi(n+1)^{2 s+1} \varrho_{0}}{2^{2(s+1) n+1}}, \\
\alpha_{j i}=\frac{\pi(n+1)^{2 s+1}}{2^{2(s+1) n+1}} \sum_{\ell=\left[\frac{i+1}{2}\right]}^{p} \frac{\left(1-\xi_{j}^{2}\right)^{\ell} b_{2 \ell-i, j, 2 \ell}}{(i-1) ! 2^{2 \ell} \ell(n+1)^{2 \ell}} \sum_{k=0}^{\ell}\left(\begin{array}{c}
2 \ell \\
\ell-k
\end{array}\right) \varrho_{k},
\end{gathered}
$$

where $b_{k, j, \ell}=\frac{1}{k !}\left(L_{j}(t)^{-\ell}\right)_{t=\xi_{j}}^{(k)}$ for $k \in \mathbb{N}_{0}, j=1, \ldots, n+1, \ell \in \mathbb{N}$,

$$
L_{j}(t)=\frac{\hat{T}_{n+1}(t)}{\left(t-\xi_{j}\right) \hat{T}_{n+1}^{\prime}\left(\xi_{j}\right)}, \quad j=1, \ldots, n+1,
$$

and $\varrho_{k}$ are the coefficients from the Fourier-Chebyshev series of the form

$$
\hat{\omega}_{n, s}(t) \sqrt{1-t^{2}}=\left(\frac{n+1}{2^{n}}\right)^{2 s+2} \sum_{k=0}^{\infty} \varrho_{k} T_{2 k(n+1)}(t),
$$

where the convergence holds w.r.t. the weighted $L^{1}$-norm $\int_{-1}^{1}|f(t)|\left(1-t^{2}\right)^{-1 / 2} d t$. The prime on the summations indicates that the first term is halved.

Finally, the points $\eta_{m}, m=1, \ldots, n$, in (4.10), as the zeros of $\hat{U}_{n}$, are given by $\eta_{m}=\cos (m \pi /(n+1)), m=1, \ldots, n$. Some numerical examples of calculating the coefficients $\alpha_{j i}$ from (4.9) by [45, Eq. (3.5)] can be found in [31.

Consider now the calculation of the integral

$$
I=I_{n}=\int_{-1}^{1} \sqrt{1-t^{2}} \hat{U}_{n}(t) f(t) d t \quad(n \in \mathbb{N}),
$$


TABLE 1. The coefficients $\alpha_{j i}$ from (4.11) when $n=5$ and $p=1$.

\begin{tabular}{|c|c|}
\hline$j$ & $\alpha_{j 0}$ \\
\hline \hline 1 & $.2556634646476068716196812649153241279457331867981 \mathrm{e}-3$ \\
\hline 2 & $.2556634646476068716196812649153241279457331867981 \mathrm{e}-3$ \\
\hline 3 & $.2556634646476068716196812649153241279457331867981 \mathrm{e}-3$ \\
\hline 4 & $.2556634646476068716196812649153241279457331867981 \mathrm{e}-3$ \\
\hline 5 & $.2556634646476068716196812649153241279457331867981 \mathrm{e}-3$ \\
\hline 6 & $.2556634646476068716196812649153241279457331867981 \mathrm{e}-3$ \\
\hline \hline$j$ & $\alpha_{j 1}$ \\
\hline \hline 1 & $-.8574720254918946739677216669779694286924422719574 \mathrm{e}-6$ \\
\hline 2 & $-.6277130887290624331743990566198128168479950077446 \mathrm{e}-6$ \\
\hline 3 & $-.2297589367628322407933226103581566118444472642128 \mathrm{e}-6$ \\
\hline 4 & $.2297589367628322407933226103581566118444472642128 \mathrm{e}-6$ \\
\hline 5 & $.6277130887290624331743990566198128168479950077446 \mathrm{e}-6$ \\
\hline 6 & $.8574720254918946739677216669779694286924422719574 \mathrm{e}-6$ \\
\hline \hline$j$ & $\alpha_{j} 2$ \\
\hline \hline 1 & $.5946598861672669341073580096439351481276194639361 \mathrm{e}-7$ \\
\hline 2 & $.4438601816798730410063910849224377221280090048578 \mathrm{e}-6$ \\
\hline 3 & $.8282543747430193886020463688804819294432560633220 \mathrm{e}-6$ \\
\hline 4 & $.8282543747430193886020463688804819294432560633220 \mathrm{e}-6$ \\
\hline 5 & $.4438601816798730410063910849224377221280090048578 \mathrm{e}-6$ \\
\hline 6 & $.5946598861672669341073580096439351481276194639361 \mathrm{e}-7$ \\
\hline
\end{tabular}

by (4.11), where $f(t)=e^{t}$. Let $p=1$, for the sake of simplicity. By using the quoted formulas, we obtain, for $j=1, \ldots, n+1$,

$\alpha_{j 0}=\frac{(n+1) \pi}{2^{2 n+1}} \varrho_{0}, \quad \alpha_{j 1}=\frac{\pi\left(1-\xi_{j}^{2}\right) b_{1, j, 2}}{2^{2 n+3}(n+1)}\left(\varrho_{0}+\varrho_{1}\right), \quad \alpha_{j 2}=\frac{\pi\left(1-\xi_{j}^{2}\right)}{2^{2 n+3}(n+1)}\left(\varrho_{0}+\varrho_{1}\right)$,

where

$$
b_{0, j, 2}=1, \quad b_{1, j, 2}=-2 \sum_{k=1, k \neq j}^{n+1} \frac{1}{\xi_{j}-\xi_{k}} ; \quad \varrho_{0}=\frac{1}{(n+1)^{2}}, \quad \varrho_{0}+\varrho_{1}=\frac{1}{2(n+1)^{2}} .
$$

For $f(t)=e^{t}, t \in[-1,1]$, and $t \notin\left\{\eta_{1}, \eta_{2}, \ldots, \eta_{n}\right\}$, we use the well-known formula

$$
f\left[\eta_{1}, \ldots, \eta_{n}, t\right]=\sum_{k=1}^{n} \frac{e^{\eta_{k}}}{\hat{U}_{n}^{\prime}\left(\eta_{k}\right)\left(\eta_{k}-t\right)}+\frac{e^{t}}{\hat{U}_{n}(t)},
$$

in order to derive the expressions for $h_{i}=f^{(i)}\left[\eta_{1}, \ldots, \eta_{n}, \xi_{j}\right], i \leq 2$. Thus,

$$
\begin{aligned}
& h_{1}=\sum_{k=1}^{n} \frac{e^{\eta_{k}}}{\hat{U}_{n}^{\prime}\left(\eta_{k}\right)\left(\eta_{k}-\xi_{j}\right)^{2}}+\frac{e^{\xi_{j}}}{\hat{U}_{n}\left(\xi_{j}\right)}-\frac{e^{\xi_{j}} \hat{U}_{n}^{\prime}\left(\xi_{j}\right)}{\hat{U}_{n}^{2}\left(\xi_{j}\right)}, \\
& h_{2}=\sum_{k=1}^{n} \frac{e^{\eta_{k}}}{\hat{U}_{n}^{\prime}\left(\eta_{k}\right)\left(\eta_{k}-\xi_{j}\right)^{3}}+\frac{e^{\xi_{j}}}{\hat{U}_{n}\left(\xi_{j}\right)}-\frac{e^{\xi_{j}}\left(2 \hat{U}_{n}^{\prime}\left(\xi_{j}\right)+\hat{U}_{n}^{\prime \prime}\left(\xi_{j}\right)\right)}{\hat{U}_{n}^{2}\left(\xi_{j}\right)}+\frac{2 e^{\xi_{j}} \hat{U}_{n}^{\prime}\left(\xi_{j}\right)^{2}}{\hat{U}_{n}^{3}\left(\xi_{j}\right)} .
\end{aligned}
$$

In Table 1 the coefficients $\alpha_{j i}$ from (4.11) when $n=5$ and $p=1$ are displayed.

We calculated the integral $I=I_{n}$ by the quadrature formula in Corollary 3.10 from [3] (the values $I_{n}(B)$ ) and by (4.11) (the values $I_{n}(Q)$ ), for $p=1$ and $n=$ 
TABLE 2 . The values of $I_{n}$ computed by $I_{n}(Q)$, for some values of $n$.

\begin{tabular}{|c|c|}
\hline$n$ & $I_{n}(Q)$ \\
\hline \hline 8 & $6.094870483400303 \mathrm{e}-10$ \\
\hline 10 & $4.214999205369452 \mathrm{e}-13$ \\
\hline 20 & $5.939219550071948 \mathrm{e}-31$ \\
\hline 30 & $5.176695099972224 \mathrm{e}-51$ \\
\hline 40 & $1.601990967471637 \mathrm{e}-72$ \\
\hline 50 & $4.093862931060132 \mathrm{e}-95$ \\
\hline
\end{tabular}

$2,4,6$. The exact digits in $I_{n}(B)$ and $I_{n}(Q)$ are underlined.

$$
\begin{aligned}
I_{2} & =5.223312066295347966 \ldots \mathrm{e}-2 \\
I_{2}(B) & =\underline{5.221557556 \ldots \mathrm{e}-2} \\
I_{2}(Q) & =\underline{5.223312066295211333 \ldots \mathrm{e}-2} \\
I_{4} & =2.66508330148350987834889652789614 \ldots \mathrm{e}-4 \\
I_{4}(B) & =\underline{2.6650833004654 \ldots \mathrm{e}-4} \\
I_{4}(Q) & =\underline{2.66508330148350987834889652770 \ldots \mathrm{e}-4} \\
I_{6} & =5.495100894749276729283075957798070370281965375170 \ldots \mathrm{e}-7 \\
I_{6}(B) & =\underline{5.49510089474927643322 \ldots \mathrm{e}-7} \\
I_{6}(Q) & =\underline{5.4951008947492767292830759577980703702819653654 \ldots \mathrm{e}-7}
\end{aligned}
$$

The values of $I_{n}$ computed by $I_{n}(Q)$, for some values of $n$, are given in Table 2 , The same values are computed and by $I_{n}(B)$.

In conclusion, let us mention that we have used the traditional way of naming the Gauss-Kronrod quadratures, although it would have been better to use the name Gauss-Kronrod-Skutsch quadratures (see [11] for details).

\section{ACKNOWLEDGMENTS}

We are indebted to the referee for the careful reading of the manuscript and his suggestions that have improved the paper.

\section{REFERENCES}

[1] S. Bernstein, Sur les polynomes orthogonaux relatifs à un segment fini, J. Math. Pures Appl. 9 (1930), 127-177.

[2] Borislav Bojanov, On a quadrature formula of Micchelli and Rivlin, J. Comput. Appl. Math. 70 (1996), no. 2, 349-356, DOI 10.1016/0377-0427(95)00206-5. MR.1399878 (97d:41031)

[3] Borislav Bojanov and Guergana Petrova, Quadrature formulas for Fourier coefficients, J. Comput. Appl. Math. 231 (2009), no. 1, 378-391, DOI 10.1016/j.cam.2009.02.097. MR2532677 (2010e:65031)

[4] D. Calvetti, G. H. Golub, W. B. Gragg, and L. Reichel, Computation of Gauss-Kronrod quadrature rules, Math. Comp. 69 (2000), no. 231, 1035-1052, DOI 10.1090/S0025-5718-0001174-1. MR1677474 (2000j:65035)

[5] Lyubomir Čakalov, General quadrature formulas of Gaussian type, Bŭlgar. Akad. Nauk. Izv. Mat. Inst. 1 (1954), no. 2, 67-84 (Bulgarian, with Russian summary). MR0069232(16,1005d)

[6] Sven Ehrich, On stratified extensions of Gauss-Laguerre and Gauss-Hermite quadrature formulas, Proceedings of the 9th International Congress on Computational and Applied Mathematics (Leuven, 2000), 2002, pp. 291-299, DOI 10.1016/S0377-0427(01)00407-1. MR.1934445 (2003g:65027) 
[7] H. Engels, Numerical quadrature and cubature, Academic Press Inc. [Harcourt Brace Jovanovich Publishers], London, 1980. Computational Mathematics and Applications. MR.587486 (83g:65002)

[8] Walter Gautschi, Gauss-Kronrod quadrature - a survey, Numerical methods and approximation theory, III (Niš, 1987), Univ. Niš, Niš, 1988, pp. 39-66. MR960329 (89k:41035)

[9] Walter Gautschi, On the remainder term for analytic functions of Gauss-Lobatto and Gauss-Radau quadratures, Proceedings of the U.S.-Western Europe Regional Conference on Padé Approximants and Related Topics (Boulder, CO, 1988), 1991, pp. 209-226, DOI 10.1216/rmjm/1181073004. MR/1113924(93a:41071a)

[10] Walter Gautschi, Orthogonal polynomials: computation and approximation, Numerical Mathematics and Scientific Computation, Oxford University Press, New York, 2004. Oxford Science Publications. MR2061539 (2005e:42001)

[11] Walter Gautschi, A historical note on Gauss-Kronrod quadrature, Numer. Math. 100 (2005), no. 3, 483-484, DOI 10.1007/s00211-005-0592-7. MR2195449

[12] Walter Gautschi and Gradimir V. Milovanović, s-orthogonality and construction of GaussTurán-type quadrature formulae, J. Comput. Appl. Math. 86 (1997), no. 1, 205-218, DOI 10.1016/S0377-0427(97)00156-8. Special issue dedicated to William B. Gragg (Monterey, CA, 1996). MR 1491435 (99a:65030)

[13] Walter Gautschi and Sotorios E. Notaris, Gauss-Kronrod quadrature formulae for weight functions of Bernstein-Szegö type, J. Comput. Appl. Math. 25 (1989), no. 2, 199-224, DOI 10.1016/0377-0427(89)90047-2. MR988057(90d:65045)

[14] A. Ghizzetti and A. Ossicini, Quadrature formulae, Academic Press, New York, 1970. MR0269116 (42 \#4012)

[15] Aldo Ghizzetti and Alessandro Ossicini, Sull'esistenza e unicità delle formule di quadratura gaussiane, Rend. Mat. (6) 8 (1975), 1-15 (Italian, with English summary). Collection of articles dedicated to Mauro Picone on the occasion of his ninetieth birthday. MR 0380216 (52 \#1116)

[16] G. H. Golub and J. Kautský, Calculation of Gauss quadratures with multiple free and fixed knots, Numer. Math. 41 (1983), no. 2, 147-163, DOI 10.1007/BF01390210. MR703119 (84i:65030)

[17] Laura Gori and Charles A. Micchelli, On weight functions which admit explicit Gauss-Turán quadrature formulas, Math. Comp. 65 (1996), no. 216, 1567-1581, DOI 10.1090/S0025-571896-00769-7. MR 1361808 (97c:41034)

[18] L. Gori, M. L. Lo Cascio, and G. V. Milovanović, The $\sigma$-orthogonal polynomials: a method of construction, Orthogonal polynomials and their applications (Erice, 1990), IMACS Ann. Comput. Appl. Math., vol. 9, Baltzer, Basel, 1991, pp. 281-285. MR1270246

[19] D. K. Kahaner and G. Monegato, Nonexistence of extended Gauss-Laguerre and GaussHermite quadrature rules with positive weights, Z. Angew. Math. Phys. 29 (1978), no. 6, 983986, DOI 10.1007/BF01590820 (English, with German summary). MR523866 (80d:65034)

[20] A. S. Kronrod, Integration with control of accuracy, Soviet Physics Dokl. 9 (1964), 17-19. MR0199960 (33 \#8100)

[21] András Kroó and Franz Peherstorfer, Asymptotic representation of $L_{p}$-minimal polynomials, $1<p<\infty$, Constr. Approx. 25 (2007), no. 1, 29-39, DOI 10.1007/s00365-006-0628-5. MR2263735 (2007i:41005)

[22] Dirk P. Laurie, Anti-Gaussian quadrature formulas, Math. Comp. 65 (1996), no. 214, 739747, DOI 10.1090/S0025-5718-96-00713-2. MR1333318(96m:65026)

[23] Dirk P. Laurie, Calculation of Gauss-Kronrod quadrature rules, Math. Comp. 66 (1997), no. 219, 1133-1145, DOI 10.1090/S0025-5718-97-00861-2. MR1422788 (98m:65030)

[24] S. Li, Kronrod extension of Turán formula, Studia Sci. Math. Hungar. 29 (1994), no. 1-2, 71-83. MR 1283377(95j:41046)

[25] Gradimir V. Milovanović, Quadratures with multiple nodes, power orthogonality, and moment-preserving spline approximation, J. Comput. Appl. Math. 127 (2001), no. 1-2, 267286, DOI 10.1016/S0377-0427(00)00500-8. Numerical analysis 2000, Vol. V, Quadrature and orthogonal polynomials. MR1808578(2002e:65039)

[26] Gradimir V. Milovanović and Miodrag M. Spalević, Error bounds for Gauss-Turán quadrature formulae of analytic functions, Math. Comp. 72 (2003), no. 244, 1855-1872 (electronic), DOI 10.1090/S0025-5718-03-01544-8. MR.1986808(2004c:41068) 
[27] G. V. Milovanović and M. M. Spalević, An error expansion for some Gauss-Turán quadratures and $L^{1}$-estimates of the remainder term, BIT 45 (2005), no. 1, 117-136, DOI 10.1007/s10543005-2643-y. MR2164228(2006e:41058)

[28] G. V. Milovanović, M. M. Spalević, and A. S. Cvetković, Calculation of Gaussian-type quadratures with multiple nodes, Math. Comput. Modelling 39 (2004), no. 2-3, 325-347, DOI 10.1016/S0895-7177(04)90014-3. MR2037399 (2005g:65041)

[29] Gradimir V. Milovanović, Miodrag M. Spalević, and Miroslav S. Pranić, Maximum of the modulus of kernels in Gauss-Turán quadratures, Math. Comp. 77 (2008), no. 262, 985-994, DOI 10.1090/S0025-5718-07-02032-7. MR2373188 (2008k:41042)

[30] Gradimir V. Milovanović, Miodrag M. Spalević, and Miroslav S. Pranić, Error estimates for Gauss-Turán quadratures and their Kronrod extensions, IMA J. Numer. Anal. 29 (2009), no. 3, 486-507, DOI 10.1093/imanum/drm040. MR2520156 (2010e:65040)

[31] Gradimir V. Milovanović, Miodrag M. Spalević, and Miroslav S. Pranić, Bounds of the error of Gauss-Turán-type quadratures. II, Appl. Numer. Math. 60 (2010), no. 1-2, 1-9, DOI 10.1016/j.apnum.2009.08.002. MR.2566073 (2011a:65059)

[32] Giovanni Monegato, Stieltjes polynomials and related quadrature rules, SIAM Rev. 24 (1982), no. 2, 137-158, DOI 10.1137/1024039. MR652464 (83d:65067)

[33] Giovanni Monegato, An overview of the computational aspects of Kronrod quadrature rules, Numer. Algorithms 26 (2001), no. 2, 173-196, DOI 10.1023/A:1016640617732. MR.1829797 (2002a:65051)

[34] A. Ossicini and F. Rosati, Funzioni caratteristiche nelle formule di quadratura gaussiane con nodi multipli, Boll. Un. Mat. Ital. (4) 11 (1975), no. 3, suppl., 224-237 (Italian, with French summary). Collection of articles dedicated to Giovanni Sansone on the occasion of his eighty-fifth birthday. MR0408212(53 \#11977)

[35] Franz Peherstorfer, Gauss-Turán quadrature formulas: asymptotics of weights, SIAM J. Numer. Anal. 47 (2009), no. 4, 2638-2659, DOI 10.1137/070695794. MR2525614(2011d:41048)

[36] Franz Peherstorfer and Knut Petras, Ultraspherical Gauss-Kronrod quadrature is not possible for $\lambda>3$, SIAM J. Numer. Anal. 37 (2000), no. 3, 927-948 (electronic), DOI 10.1137/S0036142998327744. MR1749243 (2001g:33010)

[37] Franz Peherstorfer and Knut Petras, Stieltjes polynomials and Gauss-Kronrod quadrature for Jacobi weight functions, Numer. Math. 95 (2003), no. 4, 689-706, DOI 10.1007/s00211-0020412-2. MR2013124(2004j:33010)

[38] Tiberiu Popoviciu, Sur une généralisation de la formule d'intégration numérique de Gauss, Acad. R. P. Romîne. Fil. Iaşi. Stud. Cerc. Şti. 6 (1955), 29-57 (Romanian, with Russian and French summaries). MR0085604 (19,64h)

[39] Miodrag M. Spalević, On generalized averaged Gaussian formulas, Math. Comp. 76 (2007), no. 259, 1483-1492 (electronic), DOI 10.1090/S0025-5718-07-01975-8. MR2299784 (2008g:65047)

[40] Miodrag M. Spalević, A note on generalized averaged Gaussian formulas, Numer. Algorithms 46 (2007), no. 3, 253-264, DOI 10.1007/s11075-007-9137-8. MR2374184 (2008i:65045)

[41] Y. G. Shi, Generalized Gaussian Kronrod-Turán quadrature formulas, Acta Sci. Math. (Szeged) 62 (1996), no. 1-2, 175-185. MR1412926(97e:41068)

[42] Ying Guang Shi, Christoffel type functions for $m$-orthogonal polynomials, J. Approx. Theory 137 (2005), no. 1, 57-88, DOI 10.1016/j.jat.2005.07.007. MR2179623 (2006j:42038)

[43] Ying Guang Shi and Guoliang Xu, Construction of $\sigma$-orthogonal polynomials and Gaussian quadrature formulas, Adv. Comput. Math. 27 (2007), no. 1, 79-94, DOI 10.1007/s10444-0079033-8. MR 2317922 (2008c:65069)

[44] P. Turán, On the theory of the mechanical quadrature, Acta Sci. Math. Szeged 12 (1950), Leopoldo Fejer et Frederico Riesz LXX annos natis dedicatus, Pars A, 30-37. MR.0036797 $(12,164 \mathrm{~b})$

[45] Shijun Yang, On a quadrature formula of Gori and Micchelli, J. Comput. Appl. Math. 176 (2005), no. 1, 35-43, DOI 10.1016/j.cam.2004.07.031. MR2108100(2005k:41093) 
Mathematical Institute, Serbian Academy of Sciences and Arts, Kneza Mihaila 36, 11000 Belgrade, Serbia

E-mail address: gvm@mi.sanu.ac.rs

Department of Mathematics, University of Beograd, Faculty of Mechanical Engineering, Kraljice Marije 16, 11120 Belgrade 35, Serbia

E-mail address: mspalevic@mas.bg.ac.rs 